\title{
Separation conditions on controlled Moran constructions
}

\author{
by
}

\begin{abstract}
Antti Käenmäki and Markku Vilppolainen (Jyväskylä)
\end{abstract}
\begin{abstract}
It is well known that the open set condition and the positivity of the $t$-dimensional Hausdorff measure are equivalent on self-similar sets, where $t$ is the zero of the topological pressure. We prove an analogous result for a class of Moran constructions and we study different kinds of Moran constructions in this respect.
\end{abstract}

1. Introduction. The widely studied class of self-similar sets was introduced by Hutchinson [15]. A mapping $\varphi: \mathbb{R}^{d} \rightarrow \mathbb{R}^{d}$ is called a similitude mapping if there is $s>0$ such that $|\varphi(x)-\varphi(y)|=s|x-y|$ for all $x, y \in \mathbb{R}^{d}$. If the similitude mappings $\varphi_{1}, \ldots, \varphi_{k}$ are contractive, that is, all the Lipschitz constants are strictly less than one, then a nonempty compact set $E \subset \mathbb{R}^{d}$ is called self-similar provided that

$$
E=\varphi_{1}(E) \cup \cdots \cup \varphi_{k}(E) .
$$

From this, one can easily see that $E$ consists of smaller and smaller pieces which are geometrically similar to $E$. However, the self-similar structure is hard to recognize if these pieces overlap too much. Hutchinson [15] used a separation condition which guarantees that we can distinguish the pieces. The idea goes back to Moran [31] who studied similar constructions but without mappings. In the open set condition, it is required that there exists an open set $V$ such that all the images $\varphi_{i}(V)$ are pairwise disjoint and contained in $V$. Lalley [21] used a stronger version of the condition: in the strong open set condition, it is required that the open set $V$ above can be chosen such that $V \cap E \neq \emptyset$.

Assuming the open set condition, Hutchinson [15, §5.3] proved that the $t$-dimensional Hausdorff measure $\mathcal{H}^{t}$ of $E$ is positive, where $t$ is the zero of the so-called topological pressure. See also Moran [31, Theorem III] for

2000 Mathematics Subject Classification: Primary 28A80; Secondary 37C45.

Key words and phrases: Moran construction, iterated function system, limit set, invariant set, separation condition, open set condition, Hausdorff measure, Hausdorff dimension.

AK acknowledges the support of the Academy of Finland (project \#114821). 
the corresponding theorem for the Moran constructions. Schief [34, Theorem 2.1] showed, extending the ideas of Bandt and Graf [2], that the open set condition is not only sufficient but also necessary for the positivity of the Hausdorff measure. In fact, he proved that $\mathcal{H}^{t}(E)>0$ implies the strong open set condition. Later, Peres, Rams, Simon, and Solomyak [33, Theorem 1.1] showed that this equivalence also holds for self-conformal sets. See also Fan and Lau [11], Lau, Rao and Ye [22], and Ye [40]. Observe that in these results, it is essential to have a finite number of mappings. Szarek and Wędrychowicz [35] have shown that in the infinite case the open set condition does not necessarily imply the strong open set condition.

The main theme in this article is to study the relationship between separation conditions and the Hausdorff measure on limit sets of Moran constructions. More precisely, we study what can be said about Schief's result in this setting. Since the open set condition requires the use of mappings, we introduce a representative form for it to be used on Moran constructions. We also study invariant sets of certain iterated function systems without assuming conformality. We generalize many classical results into these settings.

The article is organized as follows. In Section 2, we introduce the concept of semiconformal measure on the symbol space and prove the existence of such measures. The projection of a semiconformal measure onto the limit set of a Moran construction gives us valuable information about the limit set provided that the construction is properly controlled and the pieces used in the construction are appropriately separated. We introduce the definitions of the controlled Moran construction (CMC) and suitable separation conditions in Section 3. We also specify a class of CMC's, the so called tractable CMC's, for which a natural separation condition is equivalent to the positivity of $\mathcal{H}^{t}(E)$, where $E$ is the limit set and $t$ the zero of the topological pressure. In Section 4, we consider a subclass of tractable CMC's, which we call semiconformal CMC's. We show that this class has properties that allow us to consider it as a natural replacement of the class of conformal iterated function systems to the setting of Moran constructions. Without the assumption of conformality, we study separation conditions on iterated function systems in Section 5. The last section is devoted to examples.

2. Semiconformal measure. In this section, we work only in the symbol space. We begin by fixing some notation to be used throughout this article. As usual, let $I$ be a finite set with at least two elements. Put $I^{*}=$ $\bigcup_{n=1}^{\infty} I^{n}$ and $I^{\infty}=I^{\mathbb{N}}$. Now for each $i \in I^{*}$, there is $n \in \mathbb{N}$ such that $i=\left(i_{1}, \ldots, i_{n}\right) \in I^{n}$. We call this $n$ the length of $i$ and we write $|i|=n$. The length of elements in $I^{\infty}$ is infinity. Moreover, if $i \in I^{*}$ and $j \in I^{*} \cup I^{\infty}$, then $i j$ denotes the element obtained by juxtaposing $i$ and $j$. For $i \in I^{*}$ and $A \subset I^{\infty}$, we define $[\mathrm{i} ; A]=\{\mathrm{ij}: \mathrm{j} \in A\}$ and we call $[\mathrm{i}]=\left[\mathrm{i} ; I^{\infty}\right]$ a cylinder 
set of level $|\mathrm{i}|$. If $\mathrm{j} \in I^{*} \cup I^{\infty}$ and $1 \leq n<|j|$, we define $\left.\mathrm{j}\right|_{n}$ to be the unique $i \in I^{n}$ for which $j \in[i]$. We also set $i^{-}=\left.i\right|_{|i|-1}$. The notation $i \perp j$ means that $i, j \in I^{*}$ are incomparable, that is, $[i] \cap[j]=\emptyset$. We call a set $A \subset I^{*}$ incomparable if all of its elements are mutually incomparable. Finally, $i \wedge j$ is the common beginning of $i \in I^{*}$ and $j \in I^{*}$, that is, $i \wedge j=\left.i\right|_{n}=\left.j\right|_{n}$, where $n=\min \left\{k-1:\left.\mathrm{i}\right|_{k} \neq\left.\mathrm{j}\right|_{n}\right\}$.

Defining

$$
|i-j|= \begin{cases}2^{-|i \wedge j|}, & i \neq j \\ 0, & i=j\end{cases}
$$

for $\mathrm{i}, \mathrm{j} \in I^{\infty}$ makes $\left(I^{\infty},|\cdot|\right)$ a compact metric space. We call $\left(I^{\infty},|\cdot|\right)$ a symbol space and an element $i \in I^{\infty}$ a symbol. If there is no danger of misunderstanding, let us also call an element $i \in I^{*}$ a symbol. Define the left shift $\sigma: I^{\infty} \rightarrow I^{\infty}$ by setting

$$
\sigma\left(i_{1}, i_{2}, \ldots\right)=\left(i_{2}, i_{3}, \ldots\right) .
$$

Write $\sigma\left(i_{1}, \ldots, i_{n}\right)=\left(i_{2}, \ldots, i_{n}\right) \in I^{n-1}$. Observe that to be precise in our definitions, we need to work with "empty symbols", that is, symbols with zero length. However, this is left to the reader.

We now present sufficient conditions for the existence of the so-called semiconformal measure. Our presentation here has common points with [5] and $[4, \S 2.1 .2]$. Suppose the collection $\left\{s_{i}>0: i \in I^{*}\right\}$ satisfies the following two assumptions:

(S1) There exists a constant $D \geq 1$ such that

$$
D^{-1} s_{\mathrm{i}} s_{\mathrm{j}} \leq s_{\mathrm{ij}} \leq D s_{\mathrm{i}} s_{\mathrm{j}} \quad \text { for all } \mathrm{i}, \mathrm{j} \in I^{*} \text {. }
$$

(S2) $\max _{\mathrm{i} \in I^{n}} s_{\mathrm{i}} \rightarrow 0$ as $n \rightarrow \infty$.

Given $t \geq 0$, we define the topological pressure to be

$$
P(t)=\lim _{n \rightarrow \infty} \frac{1}{n} \log \sum_{i \in I^{n}} s_{i}^{t} .
$$

The limit above exists by the standard theory of subadditive sequences since

$$
\sum_{\mathbf{i} \in I^{n+m}} s_{\mathbf{i}}^{t} \leq D^{t} \sum_{\mathbf{i} \in I^{n+m}} s_{\left.\mathbf{i}\right|_{n}}^{t} s_{\sigma^{n}(\mathbf{i})}^{t}=D^{t} \sum_{\mathbf{i} \in I^{n}} s_{\mathbf{i}}^{t} \sum_{\mathbf{j} \in I^{m}} s_{j}^{t}
$$

in view of $(\mathrm{S} 1)$.

As a function, $P:[0, \infty) \rightarrow \mathbb{R}$ is convex: Let $0 \leq t_{1} \leq t_{2}$ and $\lambda \in(0,1)$. Now Hölder's inequality implies

$$
\sum_{i \in I^{n}} s_{i}^{\lambda t_{1}+(1-\lambda) t_{2}}=\sum_{i \in I^{n}}\left(s_{i}^{t_{1}}\right)^{\lambda}\left(s_{i}^{t_{2}}\right)^{1-\lambda} \leq\left(\sum_{i \in I^{n}} s_{i}^{t_{1}}\right)^{\lambda}\left(\sum_{i \in I^{n}} s_{i}^{t_{2}}\right)^{1-\lambda}
$$

from which the convexity follows. According to (S2), we may choose $n \in \mathbb{N}$ 
so that $\max _{\mathbf{i} \in I^{n}} s_{\mathbf{i}}<D^{-1}$. Then, using (S1), we have

$$
P(t) \leq \lim _{k \rightarrow \infty} \frac{1}{k n} \log \left(D^{t} \sum_{\mathrm{i} \in I^{n}} s_{\mathrm{i}}^{t}\right)^{k} \leq \frac{1}{n} \log \left(D \max _{\mathrm{i} \in I^{n}} s_{\mathrm{i}}\right)^{t}+\frac{1}{n} \log \# I^{n} .
$$

Hence $P(t) \rightarrow-\infty$ as $t \rightarrow \infty$ and, noting that $P(0)=\log \# I>0$, there exists a unique $t \geq 0$ for which $P(t)=0$.

Lemma 2.1. Suppose $t \geq 0$. Then

$$
D^{-t} e^{n P(t)} \leq \sum_{\mathbf{i} \in I^{n}} s_{\mathbf{i}}^{t} \leq D^{t} e^{n P(t)} \quad \text { for all } n \in \mathbb{N} .
$$

Proof. Since

$$
P(t) \geq \lim _{k \rightarrow \infty} \frac{1}{k n} \log \left(D^{-t} \sum_{\mathbf{i} \in I^{n}} s_{\mathrm{i}}^{t}\right)^{k}=\log \left(\sum_{\mathbf{i} \in I^{n}} s_{\mathrm{i}}^{t}\right)^{1 / n}+\log D^{-t / n}
$$

by (S2), we get $\sum_{\mathbf{i} \in I^{n}} s_{\mathbf{i}}^{t} \leq D^{t} e^{n P(t)}$ for each $n \in \mathbb{N}$. The other inequality follows similarly from $(2.1)$.

Let $l^{\infty}$ be the linear space of all bounded sequences on the real line. Recalling [32, Theorem 7.2], we say that a Banach limit is any mapping $L: l^{\infty} \rightarrow \mathbb{R}$ for which

(L1) $L$ is linear,

(L2) $L\left(\left(x_{n}\right)_{n \in \mathbb{N}}\right)=L\left(\left(x_{n+1}\right)_{n \in \mathbb{N}}\right)$,

(L3) $\liminf _{n \rightarrow \infty} x_{n} \leq L\left(\left(x_{n}\right)_{n \in \mathbb{N}}\right) \leq \lim \sup _{n \rightarrow \infty} x_{n}$.

To simplify the notation, we write $\operatorname{Lim}_{n} x_{n}=L\left(\left(x_{n}\right)_{n \in \mathbb{N}}\right)$.

We call a Borel probability measure $\mu$ on $I^{\infty} t$-semiconformal if there exists a constant $c \geq 1$ such that

$$
c^{-1} e^{-|\mathbf{i}| P(t)} s_{\mathbf{i}}^{t} \leq \mu([\mathbf{i}]) \leq c e^{-|\mathbf{i}| P(t)} s_{\dot{\mathbf{i}}}^{t} \quad \text { for all } \mathbf{i} \in I^{*} .
$$

A Borel probability measure $\mu$ on $I^{\infty}$ is called invariant if $\mu([\mathbf{i}])=\mu\left(\sigma^{-1}([\mathbf{i}])\right)$ for each $i \in I^{*}$, and ergodic if $\mu(A)=0$ or $\mu(A)=1$ for every Borel set $A \subset I^{\infty}$ for which $A=\sigma^{-1}(A)$. The Banach limit is a rather standard tool in producing an invariant measure from a given measure (for example, see [38, Corollary 1] and [28, Theorem 3.8]). In the following theorem, we construct a family of semiconformal measures by applying a Banach limit to a suitable collection of bounded set functions.

TheOREM 2.2. For each $t \geq 0$ there exists a unique invariant $t$-semiconformal measure. Furthermore, it is ergodic.

Proof. Define, for each $i \in I^{*}$ and $n \in \mathbb{N}$,

$$
\nu_{n}(\mathrm{i})=\frac{\sum_{\mathrm{j} \in I^{n}} s_{\mathrm{ij}}^{t}}{\sum_{\mathrm{j} \in I^{|i|+n}} s_{\mathrm{j}}^{t}} .
$$


Letting $\nu(\mathrm{i})=\operatorname{Lim}_{n} \nu_{n}(\mathrm{i})$ and using (L1) and (L2), we have

$$
\begin{aligned}
\sum_{j \in I} \nu(\mathrm{i} j) & =\sum_{j \in I} \operatorname{Lim}_{n} \nu_{n}(\mathrm{i} j)=\operatorname{Lim}_{n} \frac{\sum_{j \in I} \sum_{\mathrm{j} \in I^{n}} s_{\mathrm{i} j \mathrm{j}}^{t}}{\sum_{\mathrm{j} \in I^{|\mathrm{i}|+1+n}} s_{\mathrm{j}}^{t}} \\
& =\operatorname{Lim}_{n} \nu_{n+1}(\mathrm{i})=\operatorname{Lim}_{n} \nu_{n}(\mathrm{i})=\nu(\mathbf{i})
\end{aligned}
$$

for all $i \in I^{*}$. Since, by Lemma 2.1 ,

$$
\begin{aligned}
\nu_{n}(\mathbf{i}) & \leq D^{t} e^{-(|\mathbf{i}|+n) P(t)} \sum_{\mathbf{j} \in I^{n}} s_{\mathbf{i} j}^{t} \leq D^{2 t} e^{-(|\mathbf{i}|+n) P(t)} s_{\mathbf{i}}^{t} \sum_{\mathbf{j} \in I^{n}} s_{\mathbf{j}}^{t} \\
& \leq D^{3 t} e^{-|\mathbf{i}| P(t)} s_{\mathbf{i}}^{t}
\end{aligned}
$$

and similarly the other way around, we have, using (L3),

$$
D^{-3 t} e^{-|\mathbf{i}| P(t)} s_{\mathbf{i}}^{t} \leq \nu(\mathbf{i}) \leq D^{3 t} e^{-|\mathbf{i}| P(t)} s_{\mathbf{i}}^{t} .
$$

Next define, for each $i \in I^{*}$ and $n \in \mathbb{N}$,

$$
\mu_{n}(\mathrm{i})=\sum_{\mathrm{j} \in I^{n}} \nu(\mathrm{ji})
$$

Letting $\mu(\mathbf{i})=\operatorname{Lim}_{n} \mu_{n}(\mathbf{i})$, we have $\mu(\mathbf{i})>0$ and, using (2.3),

$$
\sum_{j \in I} \mu(\mathbf{i} j)=\operatorname{Lim}_{n} \sum_{j \in I} \sum_{j \in I^{n}} \nu(j \mathbf{i} j)=\mu(\mathbf{i})
$$

for all $i \in I^{*}$. Observe also that

$$
\sum_{j \in I} \mu(j \mathrm{i})=\operatorname{Lim}_{n} \sum_{j \in I} \sum_{\mathbf{j} \in I^{n}} \nu(\mathbf{j} j \mathrm{i})=\operatorname{Lim}_{n} \mu_{n+1}(\mathbf{i})=\mu(\mathbf{i})
$$

for all $i \in I^{*}$. Using now (2.4) and Lemma 2.1, we have

$$
\begin{aligned}
\mu_{n}(\mathbf{i}) & \leq D^{3 t} \sum_{\mathbf{j} \in I^{n}} e^{-|\mathbf{i}| P(t)} s_{\mathbf{i j}}^{t} \leq D^{4 t} e^{-|\mathbf{i}| P(t)} s_{\mathbf{i}}^{t} \sum_{\mathbf{j} \in I^{n}} e^{-|j| P(t)} s_{\mathbf{j}}^{t} \\
& \leq D^{5 t} e^{-|\mathbf{i}| P(t)} s_{\mathbf{i}}^{t}
\end{aligned}
$$

and similarly the other way around. Hence

$$
D^{-5 t} e^{-|\mathbf{i}| P(t)} s_{\dot{\mathbf{i}}}^{t} \leq \mu(\mathbf{i}) \leq D^{5 t} e^{-|\mathbf{i}| P(t)} s_{\dot{\mathbf{}}}^{t} .
$$

Now, identifying $i \in I^{*}$ with the cylinder [i], we notice, using (2.5), that $\mu$ is a probability measure on the semialgebra of all cylinder sets. Hence, by the Carathéodory-Hahn theorem (see [39, Theorem 11.20]), $\mu$ extends to a Borel probability measure on $I^{\infty}$. Observe that by (2.6) and (2.7), $\mu$ is an invariant $t$-semiconformal measure.

We shall next prove that $\mu$ is ergodic. We have learned the following argument from the proof of [28, Theorem 3.8]. Assume on the contrary that there exists a $\mu$-measurable set $A \subset I^{\infty}$ such that $\sigma^{-1}(A)=A$ and $0<\mu(A)<1$. 
Fix $\mathrm{i} \in I^{*}$ and take an incomparable set $R \subset I^{*}$ for which $I^{\infty} \backslash A \subset \bigcup_{\mathrm{j} \in R}[\mathrm{j}]$ and

$$
\sum_{\mathrm{j} \in R} \mu([\mathrm{ij}]) \leq 2 \mu\left(\left[\mathrm{i} ; I^{\infty} \backslash A\right]\right) .
$$

Using (2.7), we infer

$$
\begin{aligned}
\mu\left(\left[\mathrm{i} ; I^{\infty} \backslash A\right]\right) & \geq 2^{-1} D^{-6 t} s_{\mathbf{i}}^{t} \sum_{\mathbf{j} \in R} e^{-|\mathbf{i} j| P(t)} s_{\mathbf{j}}^{t} \\
& \geq 2^{-1} D^{-16 t} e^{|\mathbf{i}| P(t)} \mu([\mathbf{i}]) \sum_{\mathbf{j} \in R} e^{-|\mathbf{i} j| P(t)} e^{|\mathbf{j}| P(t)} \mu([\mathbf{j}]) \\
& \geq 2^{-1} D^{-16 t} \mu([\mathbf{i}]) \mu\left(I^{\infty} \backslash A\right) .
\end{aligned}
$$

Therefore

$$
\begin{aligned}
\mu\left(\sigma^{-n}(A) \cap[\mathbf{i}]\right) & =\mu([\mathbf{i} ; A])=\mu([\mathbf{i}])-\mu\left(\left[\mathbf{i} ; I^{\infty} \backslash A\right]\right) \\
& \leq\left(1-2^{-1} D^{-16 t} \mu\left(I^{\infty} \backslash A\right)\right) \mu([\mathbf{i}])
\end{aligned}
$$

for each $i \in I^{*}$. Define $\gamma=\left(1-2^{-1} D^{-16 t} \mu\left(I^{\infty} \backslash A\right)\right)$ and $\eta=\left(1+\gamma^{-1}\right) / 2$. Take an incomparable set $R \subset I^{*}$ for which $A \subset \bigcup_{i \in R}[\mathbf{i}]$ and $\sum_{\mathbf{i} \in R} \mu([\mathbf{i}]) \leq$ $\eta \mu(A)$. Since now, by $(2.8)$,

$$
\begin{aligned}
\mu(A) & =\sum_{\mathbf{i} \in R} \mu(A \cap[\mathbf{i}])=\sum_{\mathbf{i} \in R} \mu\left(\sigma^{-n}(A) \cap[\mathbf{i}]\right) \\
& \leq \sum_{\mathbf{i} \in R} \gamma \mu([\mathbf{i}]) \leq \gamma \eta \mu(A)<\mu(A),
\end{aligned}
$$

we have finished the proof of the ergodicity.

To prove the uniqueness, assume that $\widetilde{\mu}$ is another invariant $t$-semiconformal measure. Now there exists $c \geq 1$ such that $\widetilde{\mu}([i]) \leq c \mu([i])$ for all $i \in I^{*}$. By the uniqueness of the Carathéodory-Hahn extension, this inequality implies that also $\widetilde{\mu} \leq c \mu$. The ergodicity of $\mu$ implies that $\widetilde{\mu}=\mu$ (see [37, Theorem 6.10]).

Let us next prove two lemmas for future reference. Define, for $i \in I^{*}$,

$$
\begin{aligned}
& \Omega_{\mathrm{i}}=\left\{\mathrm{j} \in I^{\infty}: \sigma^{n-1}(\mathrm{j}) \in[\mathrm{i}] \text { for infinitely many } n \in \mathbb{N}\right\}, \\
& \Omega_{\mathrm{i}}^{0}=\left\{\mathrm{j} \in I^{\infty}: \sigma^{n-1}(\mathrm{j}) \notin[\mathrm{i}] \text { for every } n \in \mathbb{N}\right\} .
\end{aligned}
$$

Lemma 2.3. Suppose $\mu$ is an invariant ergodic Borel probability measure on $I^{\infty}$. If $\mathrm{i} \in I^{*}$ and $\mu([\mathrm{i}])>0$, then $\mu\left(\Omega_{\mathrm{i}}^{0}\right)=0$ and $\mu\left(\Omega_{\mathrm{i}}\right)=1$.

Proof. Take $\mathrm{i} \in I^{*}$ such that $\mu([\mathbf{i}])>0$. Notice that $\sigma^{-1}\left(I^{\infty} \backslash \Omega_{\mathrm{i}}^{0}\right) \subset$ $I^{\infty} \backslash \Omega_{\mathrm{i}}^{0}$ and $\mu\left(\sigma^{-1}\left(I^{\infty} \backslash \Omega_{\mathrm{i}}^{0}\right)\right)=\mu\left(I^{\infty} \backslash \Omega_{\mathrm{i}}^{0}\right)$ by the invariance of $\mu$. Since $\Omega_{\mathrm{i}}=\bigcap_{n=0}^{\infty} \sigma^{-n}\left(I^{\infty} \backslash \Omega_{\mathrm{i}}^{0}\right)$, we have $\sigma^{-1}\left(\Omega_{\mathrm{i}}\right)=\Omega_{\mathrm{i}}$, and by the ergodicity 
of $\mu$, either $\mu\left(\Omega_{\mathrm{i}}\right)=0$ or $\mu\left(\Omega_{\mathrm{i}}\right)=1$. Since

$$
\mu\left(\Omega_{\mathrm{i}}\right)=\lim _{n \rightarrow \infty} \mu\left(\sigma^{-n}\left(I^{\infty} \backslash \Omega_{\mathrm{i}}^{0}\right)\right)=\mu\left(I^{\infty} \backslash \Omega_{\mathrm{i}}^{0}\right) \geq \mu([\mathrm{i}])>0,
$$

it follows that $\mu\left(I^{\infty} \backslash \Omega_{\mathrm{i}}^{0}\right)=\mu\left(\Omega_{\mathrm{i}}\right)=1$.

Assume that $I$ has at least three elements. For a fixed $j \in I$, we set $I_{j}=I \backslash\{j\}$ and define

$$
P_{j}(t)=\lim _{n \rightarrow \infty} \frac{1}{n} \log \sum_{i \in I_{j}^{n}} s_{i}^{t} .
$$

LemmA 2.4. If $P(t)=0$ and $j \in I$ then $P_{j}(t)<0$.

Proof. Using Theorem 2.2, we denote by $\mu$ the invariant ergodic Borel probability measure on $I^{\infty}$ for which

$$
c^{-1} s_{i}^{t} \leq \mu([\dot{i}]) \leq c s_{i}^{t}
$$

for a constant $c \geq 1$ and all $i \in I^{*}$. Assume now on the contrary that there is $j \in I$ such that $P_{j}(t)=0$. Using Theorem 2.2 , we denote by $\mu_{j}$ the unique invariant $t$-semiconformal measure on $I_{j}^{\infty}$. Observe that there exists a constant $c_{j} \geq 1$ such that

$$
c_{j}^{-1} s_{\dot{i}}^{t} \leq \mu_{j}([\dot{i}]) \leq c_{j} s_{i}^{t}
$$

for all $i \in I_{j}^{*}$. Notice also that $\mu_{j}\left(I^{\infty} \backslash I_{j}^{\infty}\right)=0$ and $\mu\left(I_{j}^{\infty}\right)=0$ by Lemma 2.3. Defining $\lambda_{j}=\frac{1}{2}\left(\mu+\mu_{j}\right)$, we have, for each $i \in I_{j}^{*}$,

$$
\begin{aligned}
\lambda_{j}([\mathrm{i}]) & =\lambda_{j}\left([\mathrm{i}] \backslash I_{j}^{\infty}\right)+\lambda_{j}\left([\mathbf{i}] \cap I_{j}^{\infty}\right) \\
& =\frac{1}{2} \mu([\mathbf{i}])+\frac{1}{2} \mu_{j}([\mathbf{i}]) \leq \frac{1}{2}\left(c+c_{j}\right) s_{i}^{t},
\end{aligned}
$$

and similarly the other way around. Hence also $\lambda_{j}$ is invariant and $t$-semiconformal on $I_{j}^{\infty}$. From the uniqueness, we infer $\lambda_{j}=\mu_{j}$, and therefore

$$
1=\mu_{j}\left(I_{j}^{\infty}\right)=\lambda_{j}\left(I_{j}^{\infty}\right)=\frac{1}{2}\left(\mu+\mu_{j}\right)\left(I_{j}^{\infty}\right)=\frac{1}{2} .
$$

This contradiction finishes the proof.

3. Controlled Moran construction. The collection $\left\{X_{i} \subset \mathbb{R}^{d}\right.$ : $\left.i \in I^{*}\right\}$ of compact sets with positive diameter is called a controlled Moran construction $(C M C)$ if

(M1) $X_{\mathrm{i} i} \subset X_{\mathrm{i}}$ for all $\mathrm{i} \in I^{*}$ and $i \in I$,

(M2) there exists a constant $D \geq 1$ such that

$$
D^{-1} \leq \frac{\operatorname{diam}\left(X_{i j}\right)}{\operatorname{diam}\left(X_{i}\right) \operatorname{diam}\left(X_{j}\right)} \leq D \quad \text { for all } i, j \in I^{*},
$$

(M3) there exists $n \in \mathbb{N}$ such that

$$
\max _{i \in I^{n}} \operatorname{diam}\left(X_{\mathrm{i}}\right)<D^{-1} \text {. }
$$


Lemma 3.1. Given a $C M C$, there are constants $c>0$ and $0<\varrho<1$ such that $\max _{\mathrm{i} \in I^{n}} \operatorname{diam}\left(X_{\mathrm{i}}\right) \leq c \varrho^{n}$ for all $n \in \mathbb{N}$.

Proof. Using (M3), we find $k \in \mathbb{N}$ and $0<a<1$ such that $\operatorname{diam}\left(X_{\mathrm{i}}\right)<$ $a / D$ for every $i \in I^{k}$. Fix $n>k$, take $i \in I^{n}$ and set $i=i_{1} \cdots i_{l}$, where $l-1$ is the integer part of $n / k, \dot{i}_{j} \in I^{k}$ for $j \in\{1, \ldots, l-1\}$, and $0<\left|\dot{i}_{l}\right| \leq k$. Since now, by (M2),

$$
\begin{aligned}
\operatorname{diam}\left(X_{\mathbf{i}}\right) & \leq D^{l-1} \operatorname{diam}\left(X_{\mathbf{i}_{1}}\right) \operatorname{diam}\left(X_{\mathbf{i}_{2}}\right) \cdots \operatorname{diam}\left(X_{\mathbf{i}_{l-1}}\right) \operatorname{diam}\left(X_{\mathbf{i}_{l}}\right) \\
& \leq D^{l-1}(a / D)^{l-1} \max _{0<|\mathbf{i}| \leq k} \operatorname{diam}\left(X_{\mathbf{i}}\right) \leq a^{-1} \max _{0<|\mathbf{i}| \leq k} \operatorname{diam}\left(X_{\mathbf{i}}\right)\left(a^{1 / k}\right)^{n},
\end{aligned}
$$

the proof is finished.

Using the assumption (M1) and Lemma 3.1, we define a projection mapping $\pi: I^{\infty} \rightarrow X$ such that

$$
\{\pi(\mathrm{i})\}=\bigcap_{n=1}^{\infty} X_{\left.\mathrm{i}\right|_{n}} \quad \text { for } \mathrm{i} \in I^{\infty} .
$$

It is clear that $\pi$ is continuous. The compact set $E=\pi\left(I^{\infty}\right)$ is called the limit set (of the CMC). We define a Borel probability measure $m$ on $E$ to be $t$-semiconformal if there exists a constant $c \geq 1$ such that

$$
\begin{gathered}
c^{-1} \operatorname{diam}\left(X_{\mathrm{i}}\right)^{t} \leq m\left(X_{\mathrm{i}}\right) \leq c \operatorname{diam}\left(X_{\mathrm{i}}\right)^{t} \quad \text { for all } \mathrm{i} \in I^{*}, \\
m\left(X_{\mathrm{i}} \cap X_{\mathrm{j}}\right)=0 \quad \text { whenever } \mathrm{i} \perp \mathrm{j} .
\end{gathered}
$$

Observe that in Section 2 we defined a semiconformal measure on $I^{\infty}$. The overlapping terminology should not be confusing as it is clear from the context which definition we use. Furthermore, for each $t \geq 0$, we set

$$
P(t)=\lim _{n \rightarrow \infty} \frac{1}{n} \log \sum_{\mathbf{i} \in I^{n}} \operatorname{diam}\left(X_{\dot{1}}\right)^{t}
$$

provided that the limit exists. It follows straight from the definition that if there exists a $t$-semiconformal measure on $E$ then $P(t)=0$. Recalling Lemma 2.1, the equation $P(t)=0$ gives a natural upper bound for the Hausdorff dimension of $E$, namely $\operatorname{dim}_{\mathrm{H}}(E) \leq t$.

The following proposition provides sufficient conditions for the existence of a $t$-semiconformal measure on $E$. We say that a CMC has the bounded overlapping property if $\sup _{x \in E} \sup \left\{\# R: R \subset\left\{\mathrm{i} \in I^{*}: x \in X_{\mathrm{i}}\right\}\right.$ is incomparable $\}<\infty$. Observe that in the proposition the assumption that for all $\mathrm{i}, \mathrm{j} \in I^{*}$ and $\mathrm{h} \in I^{\infty}$ we have $\pi(\mathrm{ih}) \in X_{\mathrm{ij}}$ whenever $\pi(\mathrm{h}) \in X_{\mathrm{j}}$ is essential (see Example 6.2).

Proposition 3.2. Given a $C M C$, the limit in (3.1) exists and there is a unique $t \geq 0$ such that $P(t)=0$. Assuming $P(t)=0$, there exists an invariant ergodic Borel probability measure $\mu$ on $I^{\infty}$ and constants $c, c^{\prime}>0$ 
such that

$$
c^{-1} \operatorname{diam}\left(X_{\mathbf{i}}\right)^{t} \leq \mu([\mathbf{i}]) \leq c \operatorname{diam}\left(X_{\mathbf{i}}\right)^{t} \quad \text { for all } \mathbf{i} \in I^{*} .
$$

Set $m=\mu \circ \pi^{-1}$. Then $\mathcal{H}^{t}(A) \leq c^{\prime} m(A)$ for every m-measurable $A \subset E$. Furthermore, if in addition the $C M C$ has the bounded overlapping property and for all $\mathrm{i}, \mathrm{j} \in I^{*}$ and $\mathrm{h} \in I^{\infty}$ we have $\pi(\mathrm{ih}) \in X_{\mathrm{ij}}$ whenever $\pi(\mathrm{h}) \in X_{\mathrm{j}}$, then $m$ is a t-semiconformal measure on $E$.

Proof. According to (M2) and Lemma 3.1, the collection $\left\{\operatorname{diam}\left(X_{\mathbf{i}}\right)\right.$ : $\left.i \in I^{*}\right\}$ satisfies (S1) and (S2). The proof of the first claim is now trivial. Suppose $P(t)=0$ and denote by $\mu$ the $t$-semiconformal measure on $I^{\infty}$ associated to this collection (see Theorem 2.2). For fixed $x \in E$ and $r>0$ take $\mathrm{i}=\left(i_{1}, i_{2}, \ldots\right) \in I^{\infty}$ such that $\pi(\mathrm{i})=x$ and choose $n$ to be the smallest integer for which $X_{\left.\mathbf{i}\right|_{n}} \subset B(x, r)$, where $B(x, r)$ is the open ball centered at $x$ with radius $r$. Setting $m=\mu \circ \pi^{-1}$ and using (M2), we obtain

$$
\begin{aligned}
m(B(x, r)) & \geq m\left(X_{\left.\mathbf{i}\right|_{n}}\right) \geq \mu\left(\left[\left.\mathbf{i}\right|_{n}\right]\right) \geq c^{-1} \operatorname{diam}\left(X_{\left.\mathbf{i}\right|_{n}}\right)^{t} \\
& \geq c^{-1} D^{-t} \operatorname{diam}\left(X_{\left.\mathbf{i}\right|_{n-1}}\right)^{t} \operatorname{diam}\left(X_{i_{n}}\right)^{t} \\
& \geq c^{-1} D^{-t} \min _{i \in I} \operatorname{diam}\left(X_{i}\right)^{t} r^{t},
\end{aligned}
$$

which, according to [10, Proposition 2.2(b)], gives the second claim. Furthermore, if the bounded overlapping property holds then the proof of $[17$, Theorem 3.7] shows that

$$
m\left(X_{\mathrm{i}} \cap X_{\mathrm{j}}\right)=0 \quad \text { whenever } \mathrm{i} \perp \mathrm{j}
$$

provided that $\mu\left(\left[\dot{i} ; \pi^{-1}\left(X_{\mathrm{h}} \cap X_{\mathrm{k}}\right)\right]\right) \leq m\left(X_{\mathrm{ih}} \cap X_{\mathrm{ik}}\right)$ for all $\mathrm{i}, \mathrm{h}, \mathrm{k} \in I^{*}$. This is guaranteed by our extra assumption. Hence

$$
\begin{aligned}
m\left(X_{\mathrm{i}}\right) & =m\left(X_{\mathrm{i}} \backslash \bigcup_{\mathrm{i} \perp \mathrm{j}} X_{\mathrm{j}} \cap X_{\mathrm{i}}\right) \\
& =\mu\left(\pi^{-1}\left(X_{\mathrm{i}}\right) \backslash \bigcup_{\mathrm{i} \perp \mathrm{j}} \pi^{-1}\left(X_{\mathrm{j}} \cap X_{\mathrm{i}}\right)\right)=\mu([\mathrm{i}]),
\end{aligned}
$$

which finishes the proof of the last claim.

In the definition that follows, we introduce a natural separation condition to be used in Moran constructions. Given a CMC and $r>0$, define

$$
Z(r)=\left\{\mathbf{i} \in I^{*}: \operatorname{diam}\left(X_{\mathbf{i}}\right) \leq r<\operatorname{diam}\left(X_{\mathbf{i}^{-}}\right)\right\},
$$

and if in addition $x \in E$, set

$$
Z(x, r)=\left\{\mathrm{i} \in Z(r): X_{\mathrm{i}} \cap B(x, r) \neq \emptyset\right\} .
$$

It is often useful to know the cardinality of $Z(x, r)$. We say that a CMC has the finite clustering property if $\sup _{x \in E} \lim \sup _{r \downarrow 0} \# Z(x, r)<\infty$. Furthermore, if $\sup _{x \in E} \sup _{r>0} \# Z(x, r)<\infty$ then the CMC is said to have the uniform finite clustering property. 
Definition 3.3. We say that a CMC satisfies the ball condition if there exists a constant $0<\delta<1$ such that for each $x \in E$ there is $r_{0}>0$ such that for every $0<r<r_{0}$ there exists a set $\left\{x_{\mathrm{i}} \in \operatorname{conv}\left(X_{\mathrm{i}}\right): \mathrm{i} \in Z(x, r)\right\}$ such that the collection $\left\{B\left(x_{\mathrm{i}}, \delta r\right): \mathrm{i} \in Z(x, r)\right\}$ is disjoint. If $r_{0}>0$ above can be chosen to be infinity for every $x \in E$ then the CMC is said to satisfy the uniform ball condition. Here $\operatorname{conv}(A)$ is the convex hull of the set $A$.

We shall next prove that the (uniform) ball condition and the (uniform) finite clustering property are equivalent.

Lemma 3.4. Given a compact connected set $A \subset \mathbb{R}^{n}$ and $k \in \mathbb{N}$, there exist $x_{1}, \ldots, x_{k} \in A$ such that the collection of balls $\left\{B\left(x_{i},(2 k)^{-1} \operatorname{diam}(A)\right)\right.$ : $i \in\{1, \ldots, k\}\}$ is disjoint and

$\#\left\{i \in\{1, \ldots, k\}: B\left(x_{i},(2 k)^{-1} \operatorname{diam}(A)\right) \cap B\left(x,(2 k)^{-1} \operatorname{diam}(A)\right) \neq \emptyset\right\} \leq 2$ for every $x \in \mathbb{R}^{n}$.

Proof. Choose $y_{1}, y_{k} \in A$ such that $\left|y_{1}-y_{k}\right|=\operatorname{diam}(A)$. Denote the line going through $y_{1}$ and $y_{k}$ by $L$ and for each $i \in\{2, \ldots, k-1\}$ set $y_{i}=$ $(1-i / k) y_{1}+(i / k) y_{k} \in L$. Using the connectedness of $A$, we find for each $i \in\{1, \ldots, k\}$ a point $x_{i} \in A$ for which the inner product $\left(x_{i}-y_{i}\right) \cdot\left(y_{k}-y_{1}\right)$ is 0 .

TheOREM 3.5. A CMC satisfies the (uniform) ball condition exactly when it has the (uniform) finite clustering property.

Proof. We shall prove the nonuniform case. The uniform case follows similarly. Assuming the ball condition, take $x \in E$ and $0<r<r_{0}$. Choose for each $i \in Z(x, r)$ a point $x_{i} \in \operatorname{conv}\left(X_{\dot{i}}\right)$ such that the balls $B\left(x_{i}, \delta r\right)$ are disjoint for all $\mathrm{i} \in Z(x, r)$. Now clearly

$$
B\left(x_{\mathrm{i}}, \delta r\right) \subset B(x,(2+\delta) r) \quad \text { for all } \mathrm{i} \in Z(x, r) .
$$

Hence

$$
\begin{aligned}
\# Z(x, r) \delta^{d} r^{d} \alpha(d) & =\sum_{\mathbf{i} \in Z(x, r)} \mathcal{H}^{d}\left(B\left(x_{\mathfrak{i}}, \delta r\right)\right)=\mathcal{H}^{d}\left(\bigcup_{\mathbf{i} \in Z(x, r)} B\left(x_{\mathfrak{i}}, \delta r\right)\right) \\
& \leq \mathcal{H}^{d}(B(x,(2+\delta) r))=(2+\delta)^{d} r^{d} \alpha(d),
\end{aligned}
$$

where $\alpha(d)$ denotes the $d$-dimensional Hausdorff measure of the unit ball. This shows that the CMC has the finite clustering property.

Conversely, by the finite clustering property, there exists $M>0$ such that for each $x \in E$ there is $r_{0}>0$ such that $\# Z(x, r)<M$ whenever $0<r<r_{0}$. Choose $\delta=(4 M D)^{-1} \min _{i \in I} \operatorname{diam}\left(X_{i}\right)$ and for fixed $x \in E$ and $0<r<r_{0}$ denote the symbols of $Z(x, r)$ by $i_{1}, \ldots, i_{n}$, where $n=\# Z(x, r)$. We shall define the points $x_{\mathbf{i}_{1}}, \ldots, x_{\mathbf{i}_{n}}$ needed in the ball condition inductively. Choose $x_{i_{1}}$ to be any point of $\operatorname{conv}\left(X_{\mathrm{i}_{1}}\right)$ and assume the points $x_{i_{1}}, \ldots, x_{i_{k}}$, where $k \leq n-1$, have already been chosen such 
that the collection of balls $\left\{B\left(x_{i_{i}}, \delta r\right): i \in\{1, \ldots, k\}\right\}$ is disjoint. Using Lemma 3.4, we find points $y_{1}, \ldots, y_{2 n} \in \operatorname{conv}\left(X_{\dot{1}_{k+1}}\right)$ such that the collection $\left\{B\left(y_{j},(4 n)^{-1} \operatorname{diam}\left(X_{\mathbf{i}_{k+1}}\right)\right): j \in\{1, \ldots, 2 n\}\right\}$ is disjoint. Since, by (M2),

$$
\delta r \leq(4 M D)^{-1} \min _{i \in I} \operatorname{diam}\left(X_{i}\right) \operatorname{diam}\left(X_{\mathrm{i}^{-}}\right) \leq(4 n)^{-1} \operatorname{diam}\left(X_{\mathrm{i}}\right)
$$

for every $\mathrm{i} \in Z(x, r)$, Lemma 3.4 also says that the balls $B\left(x_{i_{i}}, \delta r\right), i \in$ $\{1, \ldots, k\}$, can intersect at most $2 k$ of the balls $B\left(y_{j},(4 n)^{-1} \operatorname{diam}\left(X_{\mathbf{i}_{k+1}}\right)\right)$, $j \in\{1, \ldots, 2 n\}$. Hence, choosing $x_{i_{k+1}} \in\left\{y_{1}, \ldots, y_{2 n}\right\}$ such that

$$
B\left(x_{\mathfrak{i}_{k+1}},(4 n)^{-1} \operatorname{diam}\left(X_{\mathfrak{i}_{k+1}}\right)\right) \cap B\left(x_{\mathfrak{i}_{i}}, \delta r\right)=\emptyset
$$

for every $i \in\{1, \ldots, k\}$, we finish the proof.

It is evident that the bounded overlapping property does not imply the finite clustering property, and in Example 6.1 we show that the converse does not hold either. The natural condition

$\sup _{x \in E, r>0} \sup \left\{\# R: R \subset\left\{\mathbf{i} \in I^{*}: X_{\mathbf{i}} \cap B(x, r) \neq \emptyset\right.\right.$

and $\left.\operatorname{diam}\left(X_{\mathbf{1}^{-}}\right)>r\right\}$ is incomparable $\}<\infty$

clearly implies both the bounded overlapping property and the uniform finite clustering property. See also [28, Lemma 2.7]. However, we do not need this condition as under a minor technical assumption, the finite clustering property implies the bounded overlapping property.

LEMMA 3.6. If a CMC has the finite clustering property then it has the bounded overlapping property provided that

$$
X_{\mathrm{i}} \cap E=\pi([\mathrm{i}]) \quad \text { for each } \mathrm{i} \in I^{*} .
$$

Proof. Set $M=\sup _{x \in E} \lim \sup _{r \downarrow 0} \# Z(x, r)$. Fix $x \in E$ and assume that $R \subset I^{*}$ is a finite incomparable set such that $x \in X_{\mathrm{i}}$ for each $\mathrm{i} \in R$. Choose $r>0$ so small that $\# Z(x, r) \leq M$ and

$$
\min _{j \in Z(x, r)}|j|>\max _{i \in R}|\mathbf{i}| \text {. }
$$

By the assumption, $x \in \bigcap_{i \in R} \pi([\mathbf{i}])$, and hence for each $i \in R$ there exists at least one $i^{*} \in Z(x, r)$ such that $\left.i^{*}\right|_{n}=i$ for some $n \in \mathbb{N}$. The incomparability of $R$ now implies that $i^{*} \neq j^{*}$ for distinct $i, j \in R$. Consequently, $\# R \leq \# Z(x, r) \leq M$.

Let us examine how the Hausdorff measure is related to the ball condition. Bear in mind that the finite clustering property and the ball condition are equivalent.

THEOREM 3.7. If a CMC has the uniform finite clustering property and $P(t)=0$, and $m$ is the measure of Proposition 3.2, then there exist constants 
$r_{0}>0$ and $K \geq 1$ such that

$$
K^{-1} r^{t} \leq m(B(x, r)) \leq K r^{t} \quad \text { whenever } x \in E \text { and } 0<r<r_{0} .
$$

Consequently, $\operatorname{dim}_{\mathrm{H}}(E)=\operatorname{dim}_{\mathrm{M}}(E)=t$.

Proof. Suppose $P(t)=0$ and $m=\mu \circ \pi^{-1}$ is the measure of Proposition 3.2. Seeing that $\pi^{-1}(B(x, r)) \subset \bigcup_{\mathbf{i} \in Z(x, r)}[\mathbf{i}]$, for fixed $x \in E$ and $r>0$ we get

$$
\begin{aligned}
m(B(x, r)) & \leq \mu\left(\bigcup_{\mathbf{i} \in Z(x, r)}[\mathbf{i}]\right) \leq \sum_{\mathbf{i} \in Z(x, r)} \mu([\mathbf{i}]) \\
& \leq c \sum_{\mathbf{i} \in Z(x, r)} \operatorname{diam}\left(X_{\mathbf{i}}\right)^{t} \leq \# Z(x, r) c r^{t},
\end{aligned}
$$

which, together with the uniform finite clustering property and the proof of Proposition 3.2, gives the first claim.

The second claim follows immediately from [27, Theorem 5.7].

REMARK 3.8. We remark that in Theorem 3.7, the measure $m$ can be replaced with the Hausdorff measure $\left.\mathcal{H}^{t}\right|_{E}$ on recalling [10, Proposition 2.2]. In fact, it is sufficient to assume the finite clustering property instead of the uniform finite clustering property to see that $\left.\mathcal{H}^{t}\right|_{E}$ is proportional to $m$. In particular, under this assumption, $0<\mathcal{H}^{t}(E)<\infty$.

One could easily prove that if $\left.\mathcal{H}^{t}\right|_{E}$ is $t$-semiconformal then there exists a set $A \subset E$ with $\mathcal{H}^{t}(E \backslash A)=0$ such that $\sup _{x \in A} \lim \sup _{r \downarrow 0} \# Z(x, r)$ $<\infty$. Since this hardly generalizes to the whole set $E$ without any additional assumption, we propose the following definition. We say that a $\mathrm{CMC}$ is tractable if there exists a constant $C \geq 1$ such that for each $r>0$ we have

$$
\operatorname{dist}\left(X_{\mathrm{hi}}, X_{\mathrm{hj}}\right) \leq C \operatorname{diam}\left(X_{\mathrm{h}}\right) r
$$

whenever $\mathrm{h} \in I^{*}, \mathrm{i}, \mathrm{j} \in Z(r)$, and $\operatorname{dist}\left(X_{\mathrm{i}}, X_{\mathrm{j}}\right) \leq r$. See Example 6.2 for an example of a nontractable CMC. Comparing the following theorem to [34, Theorem 2.1] and [33, Theorem 1.1], we see that the uniform ball condition is a proper substitute for the open set condition in the setting of tractable CMC's. See also Example 6.4.

TheOREM 3.9. A tractable CMC has the uniform finite clustering property whenever $\mathcal{H}^{t}(E)>0$ for the unique $t \geq 0$ satisfying $P(t)=0$.

Proof. Assume on the contrary that for each $N \in \mathbb{N}$ there are $x_{N}^{\prime} \in E$ and $r_{N}^{\prime}>0$ such that $\# Z\left(x_{N}^{\prime}, r_{N}^{\prime}\right) \geq N$. For fixed $N \in \mathbb{N}$ choose $\mathrm{i} \in Z\left(x_{N}^{\prime}, r_{N}^{\prime}\right)$ so that $x_{N}^{\prime}=\pi\left(\mathrm{ik}_{0}\right)$ for some $\mathrm{k}_{0} \in I^{\infty}$. We define

$$
\Omega_{\mathrm{i}}=\left\{\mathrm{k} \in I^{\infty}: \sigma^{n-1}(\mathrm{k}) \in[\mathrm{i}] \text { for infinitely many } n \in \mathbb{N}\right\},
$$


and taking arbitrary $\mathrm{k} \in \Omega_{\mathrm{i}}$ and $n \in \mathbb{N}$ for which $\sigma^{n}(\mathrm{k}) \in[\mathrm{i}]$, we set $x=\pi(\mathrm{k})$ and $\mathrm{h}=\left.\mathrm{k}\right|_{n}$. Finally, pick $\mathrm{j}_{1}, \ldots, \mathrm{j}_{N} \in Z\left(x_{N}^{\prime}, r_{N}^{\prime}\right)$ such that $\mathrm{j}_{i} \neq \mathrm{j}_{j}$ for $i \neq j$. Since now $\operatorname{dist}\left(X_{\mathrm{i}}, X_{\mathrm{j}_{i}}\right) \leq r_{N}^{\prime}$ for every $i \in\{1, \ldots, N\}$, we have $\operatorname{dist}\left(X_{\mathrm{hi}_{\mathrm{i}}}, X_{\mathrm{hj}_{i}}\right) \leq C \operatorname{diam}\left(X_{\mathrm{h}}\right) r_{N}^{\prime}$ by assumption. Hence

$$
\begin{aligned}
\pi\left(\left[\mathrm{hj}_{i}\right]\right) & \subset X_{\mathrm{hj}_{i}} \subset B\left(x, \operatorname{diam}\left(X_{\mathrm{hi}}\right)+\operatorname{dist}\left(X_{\mathrm{hi}_{1}}, X_{\mathrm{hj}_{i}}\right)+\operatorname{diam}\left(X_{\mathrm{hj}_{i}}\right)\right) \\
& \subset B\left(x,(2 D+C) \operatorname{diam}\left(X_{\mathrm{h}}\right) r_{N}^{\prime}\right)
\end{aligned}
$$

for each $i \in\{1, \ldots, N\}$ (recall that $x \in X_{\mathrm{hi}}$ ). Therefore

$$
\pi\left(\bigcup_{i=1}^{N}\left[\mathrm{hj}_{i}\right]\right) \subset B\left(x, r_{n}\right), \quad \text { where } r_{n}=(2 D+C) \operatorname{diam}\left(X_{\left.\mathrm{k}\right|_{n}}\right) r_{N}^{\prime},
$$

and

$$
\begin{aligned}
\frac{m\left(B\left(x, r_{n}\right)\right)}{r_{n}^{t}} & \geq \frac{\sum_{i=1}^{N} \mu\left(\left[\mathrm{hj}_{i}\right]\right)}{r_{n}^{t}} \geq \frac{c^{-1} \sum_{i=1}^{N} \operatorname{diam}\left(X_{\mathrm{hj}_{i}}\right)^{t}}{r_{n}^{t}} \\
& \geq \frac{c^{-1} D^{-t} \operatorname{diam}\left(X_{\mathrm{h}}\right)^{t} \sum_{i=1}^{N} \operatorname{diam}\left(X_{\mathrm{j}_{i}}\right)^{t}}{(2 D+C)^{t} \operatorname{diam}\left(X_{\mathrm{h}}\right)^{t} r_{N}^{\prime}} \geq C_{0} N,
\end{aligned}
$$

where $\mu$ is the measure of Proposition 3.2, $m=\mu \circ \pi^{-1}$, and the constant $C_{0}>0$ does not depend on $n$ or $N$. Since $r_{n} \downarrow 0$ as $n \rightarrow \infty$, we obtain

$$
\limsup _{r \downarrow 0} \frac{m(B(x, r))}{r^{t}} \geq C_{0} N \quad \text { for all } x \in \pi\left(\Omega_{\mathbf{i}}\right),
$$

which, according to [10, Proposition 2.2(b)], gives

$$
\mathcal{H}^{t}\left(\pi\left(\Omega_{\mathrm{i}}\right)\right) \leq 2^{t} C_{0}^{-1} N^{-1} m\left(\pi\left(\Omega_{\mathrm{i}}\right)\right) .
$$

Since $1=\mu\left(\Omega_{\mathrm{i}}\right) \leq m\left(\pi\left(\Omega_{\mathrm{i}}\right)\right) \leq 1$ by Lemma 2.3 , we have, using (3.3) and Proposition 3.2,

$$
\begin{aligned}
\mathcal{H}^{t}(E) & \leq \mathcal{H}^{t}\left(\pi\left(\Omega_{\mathrm{i}}\right)\right)+\mathcal{H}^{t}\left(E \backslash \pi\left(\Omega_{\mathrm{i}}\right)\right) \\
& \leq 2^{t} C_{0}^{-1} N^{-1} m\left(\pi\left(\Omega_{\mathrm{i}}\right)\right)+c^{\prime} m\left(E \backslash \pi\left(\Omega_{\mathrm{i}}\right)\right) \leq 2^{t} C_{0}^{-1} N^{-1},
\end{aligned}
$$

which leads to a contradiction as $N \rightarrow \infty$.

To summarize the implications of the previous theorem, we finish this section with the following corollary.

Corollary 3.10. For a tractable CMC, the following are equivalent:

(1) The ball condition.

(2) The uniform ball condition.

(3) $\mathcal{H}^{t}(E)>0$ if $P(t)=0$.

(4) There exist constants $r_{0}>0$ and $K \geq 1$ such that

$$
K^{-1} r^{t} \leq\left.\mathcal{H}^{t}\right|_{E}(B(x, r)) \leq K r^{t}
$$

whenever $x \in E, 0<r<r_{0}$, and $P(t)=0$. 
4. Semiconformal Moran construction. In a tractable CMC, we require that the relative positions of the sets $X_{\dot{i}}, i \in I^{*}$, follow the rule given in (3.2). The only restriction on the shapes of these sets comes from (M2) and (M3). Assuming more on the shape, we are able to prove that the Hausdorff dimension and upper Minkowski dimension of the limit set coincide, and if the uniform ball condition is satisfied then the dimension of the intersection of incomparable cylinder sets is small. We say that a CMC is semiconformal if there is a constant $C^{*} \geq 1$ such that

$$
\frac{\operatorname{dist}\left(X_{\mathrm{hi}}, X_{\mathrm{hj}}\right)}{\operatorname{diam}\left(X_{\mathrm{h}}\right)} \leq C^{*} \frac{\operatorname{dist}\left(X_{\mathrm{ki}}, X_{\mathrm{kj}}\right)}{\operatorname{diam}\left(X_{\mathrm{k}}\right)} \quad \text { for all } \mathrm{h}, \mathrm{k}, \mathrm{i}, \mathrm{j} \in I^{*} .
$$

This property implies that the limit set is "approximately self-similar". Observe that (4.1) is equivalent to the existence of a constant $C \geq 1$ for which

$$
\begin{aligned}
C^{-1} \operatorname{diam}\left(X_{\mathrm{h}}\right) \operatorname{dist}\left(X_{\mathrm{i}}, X_{\mathrm{j}}\right) & \leq \operatorname{dist}\left(X_{\mathrm{hi}}, X_{\mathrm{hj}}\right) \\
& \leq C \operatorname{diam}\left(X_{\mathrm{h}}\right) \operatorname{dist}\left(X_{\mathrm{i}}, X_{\mathrm{j}}\right)
\end{aligned}
$$

for all $h, i, j \in I^{*}$. We notice immediately that a semiconformal CMC is tractable, which indicates, for example, that the finite clustering property and the uniform finite clustering property are equivalent.

Let us first introduce natural mappings for a semiconformal CMC.

LEMma 4.1. If a CMC is semiconformal then for each $\mathrm{i} \in I^{*}$ there exists a mapping $\varphi_{\mathrm{i}}: E \rightarrow E$ such that $\varphi_{\mathrm{i}}(\pi(\mathrm{h}))=\pi(\mathrm{ih})$ for $\mathrm{h} \in I^{\infty}$ and

$$
C^{-1} \operatorname{diam}\left(X_{\mathrm{i}}\right)|x-y| \leq\left|\varphi_{\mathrm{i}}(x)-\varphi_{\mathrm{i}}(y)\right| \leq C \operatorname{diam}\left(X_{\mathrm{i}}\right)|x-y|
$$

for all $x, y \in E$.

Proof. Fix $\mathrm{i} \in I^{*}$ and $\mathrm{h}, \mathrm{k} \in I^{\infty}$. Take $\varepsilon>0$ and using Lemma 3.1, choose $n \in \mathbb{N}$ such that $\operatorname{diam}\left(X_{\mathrm{i}\left(\left.\mathrm{h}\right|_{n}\right)}\right)+\operatorname{diam}\left(X_{\mathrm{i}\left(\left.\mathrm{k}\right|_{n}\right)}\right)<\varepsilon$. Now, using (4.2), we have

$$
\begin{aligned}
|\pi(\mathrm{ih})-\pi(\mathrm{ik})| & \leq \operatorname{diam}\left(X_{\mathrm{i}\left(\left.\mathrm{h}\right|_{n}\right)}\right)+\operatorname{dist}\left(X_{\mathrm{i}\left(\left.\mathrm{h}\right|_{n}\right)}, X_{\mathrm{i}\left(\left.\mathrm{k}\right|_{n}\right)}\right)+\operatorname{diam}\left(X_{\mathrm{i}\left(\left.\mathrm{k}\right|_{n}\right)}\right) \\
& \leq C \operatorname{diam}\left(X_{\mathrm{i}}\right) \operatorname{dist}\left(X_{\left.\mathrm{h}\right|_{n}}, X_{\left.\mathrm{k}\right|_{n}}\right)+\varepsilon \\
& \leq C \operatorname{diam}\left(X_{\mathrm{i}}\right)|\pi(\mathrm{h})-\pi(\mathrm{k})|+\varepsilon .
\end{aligned}
$$

On the other hand, choosing $n \in \mathbb{N}$ such that $\operatorname{diam}\left(X_{\left.\mathrm{h}\right|_{n}}\right)+\operatorname{diam}\left(X_{\left.\mathrm{k}\right|_{n}}\right)<\varepsilon$, we get similarly

$$
\begin{aligned}
|\pi(\mathrm{ih})-\pi(\mathrm{ik})| & \geq \operatorname{dist}\left(X_{\mathrm{i}\left(\left.\mathrm{h}\right|_{n}\right)}, X_{\mathrm{i}\left(\left.\mathrm{k}\right|_{n}\right)}\right) \\
& \geq C^{-1} \operatorname{diam}\left(X_{\mathrm{i}}\right) \operatorname{dist}\left(X_{\left.\mathrm{h}\right|_{n}}, X_{\left.\mathrm{k}\right|_{n}}\right) \\
& \geq C^{-1} \operatorname{diam}\left(X_{\mathrm{i}}\right)\left(|\pi(\mathrm{h})-\pi(\mathrm{k})|-\operatorname{diam}\left(X_{\left.\mathrm{h}\right|_{n}}\right)-\operatorname{diam}\left(X_{\left.\mathrm{k}\right|_{n}}\right)\right) \\
& \geq C^{-1} \operatorname{diam}\left(X_{\mathrm{i}}\right)|\pi(\mathrm{h})-\pi(\mathrm{k})|-C^{-1} \operatorname{diam}\left(X_{\mathrm{i}}\right) \varepsilon .
\end{aligned}
$$

The claim now follows by letting $\varepsilon \downarrow 0$ since according to (4.3), we may define a mapping $\varphi_{\mathrm{i}}: E \rightarrow E$ by setting $\varphi_{\mathrm{i}}(\pi(\mathrm{h}))=\pi(\mathrm{ih})$ for $\mathrm{h} \in I^{\infty}$. 
It follows that the measure of Proposition 3.2 is semiconformal on a semiconformal CMC with the finite clustering property, in the following sense.

LEMMA 4.2. If a semiconformal CMC has the finite clustering property and $P(t)=0$, and $m$ is the measure of Proposition 3.2, then

$$
m\left(\varphi_{\mathrm{i}}(E) \cap \varphi_{\mathrm{j}}(E)\right)=0 \quad \text { whenever } \mathrm{i} \perp \mathrm{j} .
$$

Here $\varphi_{i}, i \in I^{*}$, are the mappings of Lemma 4.1 .

Proof. Since Lemma 4.1 clearly implies that $\operatorname{diam}\left(\varphi_{\mathrm{i}}(E)\right)$ is proportional to $\operatorname{diam}\left(X_{i}\right)$, the $\mathrm{CMC}$ formed by the sets $\varphi_{i}(E), i \in I^{*}$, has the same topological pressure as the original CMC. Notice that $\operatorname{diam}(E)>0$ by the finite clustering property. By the uniqueness of the invariant semiconformal measure on $I^{\infty}$, also the semiconformal measures determined by these CMC's on $I^{\infty}$ are the same. As the finite clustering property remains satisfied in the new setting and trivially $\varphi_{i}(E) \cap E=\pi([i])$ for each $i \in I^{*}$, Lemma 3.6 implies the bounded overlapping property. By the semiconformality, it is evident that for all $\mathrm{i}, \mathrm{j} \in I^{*}$ and $\mathrm{h} \in I^{\infty}$ we have $\pi(\mathrm{ih}) \in \varphi_{\mathrm{ij}}(E)$ whenever $\pi(\mathrm{h}) \in \varphi_{\mathrm{j}}(E)$, and hence Proposition 3.2 completes the proof.

Using the mappings of Lemma 4.1, we are able to prove that the Hausdorff dimension and upper Minkowski dimension of the limit set of a semiconformal CMC coincide even without assuming the ball condition.

THEOREM 4.3. If a CMC is semiconformal and $t=\operatorname{dim}_{\mathrm{H}}(E)$ then $\operatorname{dim}_{\mathrm{M}}(E)=t$ and $\mathcal{H}^{t}(E)<\infty$.

Proof. We may assume that $\operatorname{diam}(E)>0$. Let $\varphi_{i}$, $i \in I^{*}$, be the mappings of Lemma 4.1. Notice that, by (M2), there exists a constant $\delta>0$ such that

$$
\operatorname{diam}\left(X_{\mathrm{i} i}\right) \geq \delta \operatorname{diam}\left(X_{\mathrm{i}}\right) \quad \text { for all } \mathrm{i} \in I^{*} \text { and } i \in I .
$$

Take $x_{0} \in E, \mathrm{~h} \in I^{\infty}$ such that $x_{0}=\pi(\mathrm{h})$, and $0<r<C \operatorname{diam}(E)^{2}$. Then choose $n \in \mathbb{N}$ such that $\left.\mathrm{h}\right|_{n} \in Z\left(C^{-1} \operatorname{diam}(E)^{-1} r\right)$. Since $x_{0}=\varphi_{\left.\mathrm{h}\right|_{n}}\left(\pi\left(\sigma^{n}(\mathrm{~h})\right)\right)$, we have

$$
\left|x_{0}-\varphi_{\left.\mathrm{h}\right|_{n}}(y)\right| \leq C \operatorname{diam}\left(X_{\left.\mathrm{h}\right|_{n}}\right)\left|\pi\left(\sigma^{n}(\mathrm{~h})\right)-y\right| \leq C \operatorname{diam}\left(X_{\left.\mathrm{h}\right|_{n}}\right) \operatorname{diam}(E)<r
$$

for every $y \in E$. Hence $\varphi_{\left.\mathrm{h}\right|_{n}}(E) \subset E \cap B\left(x_{0}, r\right)$. On the other hand, by (4.4),

$$
\left|\varphi_{\left.\mathrm{h}\right|_{n}}(x)-\varphi_{\left.\mathrm{h}\right|_{n}}(y)\right| \geq C^{-1} \operatorname{diam}\left(X_{\left.\mathrm{h}\right|_{n}}\right)|x-y| \geq C^{-2} \operatorname{diam}(E)^{-1} \delta r|x-y|
$$

for all $x, y \in E$. Therefore for each $x_{0} \in E$ and $0<r<C \operatorname{diam}(E)^{2}$ there is a mapping $g: E \rightarrow E \cap B\left(x_{0}, r\right)$ and a constant $a=C^{-2} \operatorname{diam}(E)^{-1} \delta$ such that

$$
|g(x)-g(y)| \geq a r|x-y|
$$

for all $x, y \in E$. The claim now follows from [8, Theorem 4]. 
The following simple proposition shows the bi-Lipschitz invariance of a semiconformal CMC. Therefore the collection of all semiconformal CMC's is sufficiently large. Observe that despite this property the geometry of the limit set may change a lot under a bi-Lipschitz map (see [26, Lemma 3.2]).

Proposition 4.4. If $\left\{X_{\mathrm{i}}: \mathrm{i} \in I^{*}\right\}$ is a semiconformal $C M C$ with $E$ as a limit set and $h: \mathbb{R}^{d} \rightarrow \mathbb{R}^{d}$ is a bi-Lipschitz mapping, then $\left\{h\left(X_{\dot{i}}\right): i \in I^{*}\right\}$ is a semiconformal CMC with $h(E)$ as a limit set.

Proof. Fix constants $a, b>0$ such that

$$
a|x-y| \leq|h(x)-h(y)| \leq b|x-y| \quad \text { for all } x, y \in X .
$$

The condition (M1) is clearly satisfied and since $a \operatorname{diam}\left(X_{\mathrm{i}}\right) \leq \operatorname{diam}\left(h\left(X_{\mathrm{i}}\right)\right)$ $\leq b \operatorname{diam}\left(X_{\mathrm{i}}\right)$ for all $\mathrm{i} \in I^{*}$ and $a \operatorname{dist}\left(X_{\mathrm{i}}, X_{\mathrm{j}}\right) \leq \operatorname{dist}\left(h\left(X_{\mathrm{i}}\right), h\left(X_{\mathrm{j}}\right)\right) \leq$ $b \operatorname{dist}\left(X_{i}, X_{j}\right)$ for all $i, j \in I^{*}$, also (M2), (M3), and (4.2) are satisfied.

Examining the method used in [34, Theorem 2.1], one easily sees the usefulness of the set

$$
\begin{aligned}
W(\mathbf{i})=\left\{\mathrm{j} \in I^{*}:\right. & \mathrm{j}^{\prime} \in Z\left(\operatorname{diam}\left(X_{\mathrm{i}^{\prime}}\right)\right) \text { and } \\
& \operatorname{dist}\left(X_{\mathrm{i}^{\prime}}, X_{\mathrm{j}^{\prime}}\right) \leq 3 \operatorname{diam}\left(X_{\mathrm{i}^{\prime}}\right), \text { where } \\
& \left.\mathbf{i}^{\prime}=\sigma^{|\mathrm{i} \wedge \mathrm{j}|}(\mathbf{i}) \text { and } \mathrm{j}^{\prime}=\sigma^{|\mathbf{i} \wedge \mathrm{j}|}(\mathrm{j})\right\}
\end{aligned}
$$

for $i \in I^{*}$. See also $[22, \S 2]$ and $[33, \S 3]$. Notice that $i \in W(i)$. The constant 3 in (4.5) is somewhat arbitrary. The reader will easily see that any constant strictly larger than 2 would suffice. Let us next prove two technical lemmas.

Lemma 4.5. Given a $C M C$, the set $W(\mathrm{i})$ is incomparable for every $\mathrm{i} \in I^{*}$. Furthermore, if $\mathrm{j} \in W(\mathrm{i})$ then

$$
D^{-3} \min _{i \in I} \operatorname{diam}\left(X_{i}\right) \operatorname{diam}\left(X_{\mathrm{i}}\right) \leq \operatorname{diam}\left(X_{\mathrm{j}}\right) \leq D^{2} \operatorname{diam}\left(X_{\mathrm{i}}\right) .
$$

Proof. Fix $i \in I^{*}$. Observe that if $i \neq j \in W(i)$ then clearly $i \perp j$. Take $\mathrm{j}, \mathrm{h} \in W(\mathrm{i})$. If $|\mathrm{j} \wedge \mathrm{i}|<|\mathrm{h} \wedge \mathrm{i}|$, we must have $\mathrm{j} \perp \mathrm{h}$ since otherwise $j=i \wedge j$, which contradicts the first observation. If $|j \wedge i|=|h \wedge i|=: k$ then $\sigma^{k}(\mathrm{j}), \sigma^{k}(\mathrm{~h}) \in Z\left(\operatorname{diam}\left(X_{\sigma^{k}(\mathrm{i})}\right)\right)$ and hence $\mathrm{j} \perp \mathrm{h}$.

To prove the second claim, fix $i \in I^{*}$, take $j \in W(i)$, and set $i^{\prime}=$ $\sigma^{|i \wedge j|}(i)$ and $j^{\prime}=\sigma^{|i \wedge j|}(j)$. Since $j^{\prime} \in Z\left(\operatorname{diam}\left(X_{i^{\prime}}\right)\right)$, we have, using (M2),

$$
\operatorname{diam}\left(X_{\mathrm{i}^{\prime}}\right) \geq \operatorname{diam}\left(X_{\mathrm{j}^{\prime}}\right) \geq D^{-1} \min _{i \in I} \operatorname{diam}\left(X_{i}\right) \operatorname{diam}\left(X_{\mathrm{i}^{\prime}}\right) .
$$

Therefore, according to (M2),

$$
\begin{aligned}
\operatorname{diam}\left(X_{\mathrm{j}}\right) & \geq D^{-1} \operatorname{diam}\left(X_{\mathrm{i} \wedge \mathrm{j}}\right) \operatorname{diam}\left(X_{\mathrm{j}^{\prime}}\right) \\
& \geq D^{-2} \min _{i \in I} \operatorname{diam}\left(X_{i}\right) \operatorname{diam}\left(X_{\mathbf{i} \wedge \mathrm{j}}\right) \operatorname{diam}\left(X_{\mathbf{i}^{\prime}}\right) \\
& \geq D^{-3} \min _{i \in I} \operatorname{diam}\left(X_{i}\right) \operatorname{diam}\left(X_{\mathbf{i}}\right)
\end{aligned}
$$


and

$$
\begin{aligned}
\operatorname{diam}\left(X_{\mathrm{j}}\right) & \leq D \operatorname{diam}\left(X_{\mathrm{i} \wedge \mathrm{j}}\right) \operatorname{diam}\left(X_{\mathrm{j}^{\prime}}\right) \\
& \leq D \operatorname{diam}\left(X_{\mathrm{i} \wedge \mathrm{j}}\right) \operatorname{diam}\left(X_{\mathrm{i}^{\prime}}\right) \leq D^{2} \operatorname{diam}\left(X_{\mathrm{i}}\right)
\end{aligned}
$$

LEMMA 4.6. If a semiconformal CMC has the finite clustering property then

$$
\sup _{i \in I^{*}} \# W(i)<\infty .
$$

Proof. Suppose $\varphi_{i}, i \in I^{*}$, are the mappings of Lemma 4.1, $P(t)=0$, and $m=\mu \circ \pi^{-1}$ is the measure of Proposition 3.2. According to Corollary 3.10 and Theorems 3.5 and 3.7, there exists a constant $K \geq 1$ such that for every $x \in E$ and $r>0$,

$$
m(B(x, r)) \leq K r^{t}
$$

Fix $i \in I^{*}$, take $j \in W(i)$, and set $i^{\prime}=\sigma^{|i \wedge j|}(i)$ and $j^{\prime}=\sigma^{|i \wedge j|}(j)$. Since $\mathrm{j} \in W(\mathrm{i})$ and $\mathrm{j}^{\prime} \in Z\left(\operatorname{diam}\left(X_{\mathrm{i}^{\prime}}\right)\right)$, we have $\operatorname{dist}\left(X_{\mathrm{i}^{\prime}}, X_{\mathrm{j}^{\prime}}\right) \leq \operatorname{diam}\left(X_{\mathrm{i}^{\prime}}\right)$ and

$$
\begin{aligned}
\operatorname{dist}\left(X_{\mathrm{i}}, X_{\mathrm{j}}\right) & \leq C \operatorname{diam}\left(X_{\mathrm{i} \wedge \mathrm{j}}\right) \operatorname{dist}\left(X_{\mathrm{i}^{\prime}}, X_{\mathrm{j}^{\prime}}\right) \\
& \leq 3 C \operatorname{diam}\left(X_{\mathrm{i} \wedge \mathrm{j}}\right) \operatorname{diam}\left(X_{\mathrm{i}^{\prime}}\right) \leq 3 C D \operatorname{diam}\left(X_{\mathrm{i}}\right) .
\end{aligned}
$$

Using Lemma 4.5, we obtain

$$
\begin{aligned}
X_{\mathrm{j}} & \subset B\left(x, \operatorname{diam}\left(X_{\mathrm{i}}\right)+3 C D \operatorname{diam}\left(X_{\mathrm{i}}\right)+\operatorname{diam}\left(X_{\mathrm{j}}\right)\right) \\
& \subset B\left(x,\left(1+3 C D+D^{2}\right) \operatorname{diam}\left(X_{\mathrm{i}}\right)\right)
\end{aligned}
$$

for all $x \in \pi([\mathbf{i}])$ and $\mathrm{j} \in W(\mathrm{i})$. Hence

$$
\begin{aligned}
m\left(\bigcup_{\mathrm{j} \in W(\mathrm{i})} X_{\mathrm{j}}\right) & \leq m\left(B\left(x,\left(1+3 C D+D^{2}\right) \operatorname{diam}\left(X_{\mathrm{i}}\right)\right)\right) \\
& \leq K\left(1+3 C D+D^{2}\right)^{t} \operatorname{diam}\left(X_{\mathrm{i}}\right)^{t} .
\end{aligned}
$$

Since, on the other hand, we have a constant $c \geq 1$ such that

$$
\begin{aligned}
m\left(\bigcup_{j \in W(i)} X_{j}\right) & \geq m\left(\bigcup_{j \in W(i)} \varphi_{j}(E)\right)=\sum_{j \in W(i)} m\left(\varphi_{j}(E)\right) \\
& \geq \sum_{j \in W(i)} \mu([j]) \geq c^{-1} \sum_{j \in W(i)} \operatorname{diam}\left(X_{j}\right)^{t} \\
& \geq \# W(i) c^{-1} D^{-3 t} \min _{i \in I} \operatorname{diam}\left(X_{i}\right)^{t} \operatorname{diam}\left(X_{\mathrm{i}}\right)^{t}
\end{aligned}
$$

using Lemmas 4.2 and 4.5, we conclude that

$$
\# W(\mathrm{i}) \leq \frac{c K D^{3 t}\left(1+3 C D+D^{2}\right)^{t}}{\min _{i \in I} \operatorname{diam}\left(X_{i}\right)^{t}} \quad \text { for all } \mathrm{i} \in I^{*}
$$

The following theorem generalizes a crucial point of [34, Theorem 2.1] to the setting of CMC's. See also [22, Theorem 3.3] and [33, §3]. 
THEOREM 4.7. If a semiconformal CMC has the finite clustering property then there are a constant $\delta>0$ and a symbol $\mathrm{h} \in I^{*}$ such that

$$
\operatorname{dist}\left(X_{\mathrm{ih}}, X_{\mathrm{jh}}\right)>\delta\left(\operatorname{diam}\left(X_{\mathrm{i}}\right)+\operatorname{diam}\left(X_{\mathrm{j}}\right)\right) \quad \text { whenever } \mathrm{i} \perp \mathrm{j} .
$$

Proof. Using Lemma 4.6, we choose $\mathrm{h} \in I^{*}$ such that $\# W(\mathrm{~h})=$ $\sup _{\mathbf{i} \in I^{*}} \# W(\mathrm{i})$. Therefore clearly

$$
\#\{\mathrm{ij}: \mathrm{j} \in W(\mathrm{~h})\}=\# W(\mathrm{~h}) \geq \# W(\mathrm{ih}) \quad \text { for all } \mathrm{i} \in I^{*} .
$$

Since it follows immediately from the definition (4.5) that $\{\mathrm{ij}: \mathrm{j} \in W(\mathrm{~h})\} \subset$ $W($ ih), we infer that

$$
W(\mathrm{ih})=\{\mathrm{ij}: \mathrm{j} \in W(\mathrm{~h})\} \quad \text { for all } \mathrm{i} \in I^{*} .
$$

Take next $i, j \in I^{*}$ such that $i \perp j$ and set $i^{\prime}=\sigma^{|i \wedge j|}(i)$ and $j^{\prime}=$ $\sigma^{|\mathrm{i} \wedge \mathrm{j}|}(\mathrm{j})$. Let $y_{\mathrm{j}^{\prime}}=\pi(\mathrm{k}) \in X_{\mathrm{j}^{\prime} \mathrm{h}}$, where $\mathrm{k} \in\left[\mathrm{j}^{\prime} \mathrm{h}\right]$, and choose $k \in \mathbb{N}$ such that $\left.\mathrm{k}\right|_{k} \in Z\left(\operatorname{diam}\left(X_{\mathrm{i}^{\prime} \mathrm{h}}\right)\right)$. Since $\left.\mathrm{k}\right|_{1}=\left.\mathrm{j}^{\prime}\right|_{1} \neq\left.\mathrm{i}^{\prime}\right|_{1}$, by (4.6) we have $\left.\mathrm{k}\right|_{k} \notin W\left(\mathrm{i}^{\prime} \mathrm{h}\right)$. Hence the definition (4.5) yields $\operatorname{dist}\left(X_{\mathrm{k} \mid}, X_{\mathrm{i}^{\prime} \mathrm{h}}\right)>3 \operatorname{diam}\left(X_{\mathrm{i}^{\prime} \mathrm{h}}\right)$. Since $y_{\mathrm{j}^{\prime}}$ is in $X_{\left.\mathrm{k}\right|_{k}}$, we also have $\operatorname{dist}\left(y_{\mathrm{j}^{\prime}}, X_{\mathrm{i}^{\prime} \mathrm{h}}\right)>3 \operatorname{diam}\left(X_{\mathrm{i}^{\prime} \mathrm{h}}\right)$. Similarly, changing the roles of $i$ and $j$ above, we find $y_{i^{\prime}} \in X_{i^{\prime} h}$ such that $\operatorname{dist}\left(y_{i^{\prime}}, X_{j^{\prime} h}\right)>$ $3 \operatorname{diam}\left(X_{j^{\prime} \mathrm{h}}\right)$. This implies that

$$
\begin{aligned}
\left|y_{\mathrm{i}^{\prime}}-y_{\mathrm{j}^{\prime}}\right| & \geq 3 \max \left\{\operatorname{diam}\left(X_{\mathrm{i}^{\prime} \mathrm{h}}\right), \operatorname{diam}\left(X_{\mathrm{j}^{\prime} \mathrm{h}}\right)\right\} \\
& \geq \frac{3}{2}\left(\operatorname{diam}\left(X_{\mathrm{i}^{\prime} \mathrm{h}}\right)+\operatorname{diam}\left(X_{\mathrm{j}^{\prime} \mathrm{h}}\right)\right) .
\end{aligned}
$$

Since, on the other hand,

$$
\left|y_{\mathrm{i}^{\prime}}-y_{\mathrm{j}^{\prime}}\right| \leq \operatorname{diam}\left(X_{\mathrm{i}^{\prime} \mathrm{h}}\right)+\operatorname{dist}\left(X_{\mathrm{i}^{\prime} \mathrm{h}}, X_{\mathrm{j}^{\prime} \mathrm{h}}\right)+\operatorname{diam}\left(X_{\mathrm{j}^{\prime} \mathrm{h}}\right),
$$

we infer that

$$
\operatorname{dist}\left(X_{\mathrm{i}^{\prime} \mathrm{h}}, X_{\mathrm{j}^{\prime} \mathrm{h}}\right) \geq \frac{1}{2}\left(\operatorname{diam}\left(X_{\mathrm{i}^{\prime} \mathrm{h}}\right)+\operatorname{diam}\left(X_{\mathrm{j}^{\prime} \mathrm{h}}\right)\right) .
$$

Thus, by (4.2) and (M2),

$$
\begin{aligned}
\operatorname{dist}\left(X_{\mathrm{ih}}, X_{\mathrm{jh}}\right) & \geq C^{-1} \operatorname{diam}\left(X_{\mathrm{i} \wedge \mathrm{j}}\right) \operatorname{dist}\left(X_{\mathrm{i}^{\prime} \mathrm{h}}, X_{\mathrm{j}^{\prime} \mathrm{h}}\right) \\
& \geq(2 C)^{-1} \operatorname{diam}\left(X_{\mathrm{i} \wedge \mathrm{j}}\right)\left(\operatorname{diam}\left(X_{\mathrm{i}^{\prime} \mathrm{h}}\right)+\operatorname{diam}\left(X_{\mathrm{j}^{\prime} \mathrm{h}}\right)\right) \\
& \geq(2 C D)^{-1}\left(\operatorname{diam}\left(X_{\mathrm{ih}}\right)+\operatorname{diam}\left(X_{\mathrm{jh}}\right)\right) \\
& \geq\left(2 C D^{2}\right)^{-1} \operatorname{diam}\left(X_{\mathrm{h}}\right)\left(\operatorname{diam}\left(X_{\mathrm{i}}\right)+\operatorname{diam}\left(X_{\mathrm{j}}\right)\right)
\end{aligned}
$$

whenever $i \perp j$. Therefore, choosing $\delta=\left(3 C D^{2}\right)^{-1} \operatorname{diam}\left(X_{\mathrm{h}}\right)$ finishes the proof.

As a corollary, for a semiconformal Moran construction, we may choose the balls in the ball condition to be centered at $E$ and placed in such a manner that also larger collections (than required in the definition) of them are disjoint. 
COROLlary 4.8. If a semiconformal CMC satisfies the ball condition then there are a constant $\delta>0$ and a point $x \in E$ such that

$$
B\left(\varphi_{\mathrm{i}}(x), \delta \operatorname{diam}\left(X_{\mathrm{i}}\right)\right) \cap B\left(\varphi_{\mathrm{j}}(x), \delta \operatorname{diam}\left(X_{\mathrm{j}}\right)\right)=\emptyset \quad \text { whenever } \mathrm{i} \perp \mathrm{j} .
$$

Here $\varphi_{i}, i \in I^{*}$, are the mappings of Lemma 4.1 .

Proof. Let $\delta>0$ and $\mathrm{h} \in I^{*}$ be as in Theorem 4.7. Then the claim follows immediately from Theorems 3.5 and 4.7 by choosing $x \in \pi([\mathrm{h}])$.

The following improvement of Lemma 4.2 can be compared to [30, Theorem 3.3] and [23, Theorem 1.6].

Proposition 4.9. If a semiconformal CMC satisfies the ball condition then

$$
\operatorname{dim}_{\mathrm{H}}\left(\varphi_{\mathrm{i}}(E) \cap \varphi_{\mathrm{j}}(E)\right)<\operatorname{dim}_{\mathrm{H}}(E) \quad \text { whenever } \mathrm{i} \perp \mathrm{j} \text {. }
$$

Here $\varphi_{i}, i \in I^{*}$, are the mappings of Lemma 4.1 .

Proof. Let $\delta>0$ and $\mathrm{h} \in I^{*}$ be as in Theorem 4.7 and define

$$
A=\bigcup_{\mathrm{k} \in I^{*}} \varphi_{\mathrm{k}}(\pi([\mathrm{h}])) \text {. }
$$

According to Theorem 4.7, we have $\varphi_{\mathrm{i}}(\pi([\mathrm{h}])) \cap \varphi_{\mathrm{j}}(\pi([\mathrm{h}]))=\emptyset$ whenever $\mathrm{i} \perp \mathrm{j}$, and hence also $\varphi_{\mathrm{i}}(A) \cap \varphi_{\mathrm{j}}(A)=\emptyset$ if $\mathrm{i} \perp \mathrm{j}$. Thus we get

$$
\begin{aligned}
\varphi_{\mathrm{i}}(E) \cap \varphi_{\mathrm{j}}(E) & =\left(\varphi_{\mathrm{i}}(E \backslash A) \cap \varphi_{\mathrm{j}}(A)\right) \cup\left(\varphi_{\mathrm{i}}(E) \cap \varphi_{\mathrm{j}}(E \backslash A)\right) \\
& \subset \varphi_{\mathrm{i}}(E \backslash A) \cup \varphi_{\mathrm{j}}(E \backslash A)
\end{aligned}
$$

whenever $i \perp j$, from which the Lipschitz continuity implies

$$
\operatorname{dim}_{\mathrm{H}}\left(\varphi_{\mathrm{i}}(E) \cap \varphi_{\mathrm{j}}(E)\right) \leq \operatorname{dim}_{\mathrm{H}}\left(\varphi_{\mathrm{i}}(E \backslash A) \cup \varphi_{\mathrm{j}}(E \backslash A)\right) \leq \operatorname{dim}_{\mathrm{H}}(E \backslash A) .
$$

Obviously, $\left\{X_{\mathrm{i}}: \mathrm{i} \in\left(I^{|\mathrm{h}|}\right)^{*}\right\}$ is a CMC having $E$ as a limit set, whereas $E \backslash A$ is contained in the limit set $F$ of the subconstruction $\left\{X_{\mathrm{i}}: \mathrm{i} \in\left(I^{|\mathrm{h}|} \backslash\{\mathrm{h}\}\right)^{*}\right\}$. Since both these CMC's clearly have the uniform finite clustering property, Lemma 2.4 and Theorem 3.7 imply that $\operatorname{dim}_{H}(F)<\operatorname{dim}_{H}(E)$. Consequently, $\operatorname{dim}_{H}(E \backslash A)<\operatorname{dim}_{H}(E)$ and the proof is finished.

We finish this section with the following observation.

Proposition 4.10. Suppose a collection $\left\{X_{i} \subset \mathbb{R}^{d}: i \in I^{*}\right\}$ of compact sets with positive diameters satisfies the following four conditions:

(C1) $X_{\mathrm{i} i} \subset X_{\mathrm{i}}$ for all $\mathrm{i} \in I^{*}$ and $i \in I$,

(C2) there exist $\mathrm{i}, \mathrm{j} \in I^{*}$ such that $X_{\mathrm{i}} \cap X_{\mathrm{j}}=\emptyset$,

(C3) $\lim _{n \rightarrow \infty} \operatorname{diam}\left(X_{\left.\mathrm{i}\right|_{n}}\right)=0$ for every $i \in I^{\infty}$,

(C4) there exists a constant $C \geq 1$ such that for all $\mathrm{h}, \mathrm{i}, \mathrm{j} \in I^{*}$,

$$
\begin{aligned}
C^{-1} \operatorname{diam}\left(X_{\mathrm{h}}\right) \operatorname{dist}\left(X_{\mathrm{i}}, X_{\mathrm{j}}\right) & \leq \operatorname{dist}\left(X_{\mathrm{hi}}, X_{\mathrm{hj}}\right) \\
& \leq C \operatorname{diam}\left(X_{\mathrm{h}}\right) \operatorname{dist}\left(X_{\mathrm{i}}, X_{\mathrm{j}}\right) .
\end{aligned}
$$

Then the collection is a semiconformal CMC. 
Proof. It suffices to prove (M2) and (M3). To show (M2), observe first that the assumptions $(\mathrm{C} 1)$ and $(\mathrm{C} 3)$ guarantee the existence of the limit set $E$ and the claim in Lemma 4.1 follows from (C1), (C3), and (C4). Notice also that $(\mathrm{C} 2)$ implies immediately that $\operatorname{diam}(E)>0$. Let $\varphi_{\mathrm{i}}, \mathrm{i} \in I^{*}$, be the mappings of Lemma 4.1. Then

$$
\operatorname{diam}\left(\varphi_{\mathbf{i}}(E)\right) \geq\left|\varphi_{\mathbf{i}}(x)-\varphi_{\mathbf{i}}(y)\right| \geq C^{-1} \operatorname{diam}\left(X_{\mathbf{i}}\right)|x-y|
$$

for all $x, y \in E$ and it follows that

$$
\operatorname{diam}\left(X_{\mathrm{i}}\right) \leq C \operatorname{diam}(E)^{-1} \operatorname{diam}\left(\varphi_{\mathrm{i}}(E)\right)
$$

for every $i \in I^{*}$. Since

$$
\begin{aligned}
\operatorname{diam}\left(\varphi_{\mathrm{ij}}(E)\right) & =\sup _{x, y \in E}\left|\varphi_{\mathrm{i}}\left(\varphi_{\mathrm{j}}(x)\right)-\varphi_{\mathrm{i}}\left(\varphi_{\mathrm{j}}(y)\right)\right| \\
& \leq C^{2} \operatorname{diam}\left(X_{\mathrm{i}}\right) \operatorname{diam}\left(X_{\mathrm{j}}\right) \sup _{x, y \in E}|x-y|
\end{aligned}
$$

for all $i, j \in I^{*}$, we see, by (4.7), that

$$
\operatorname{diam}\left(X_{\mathrm{ij}}\right) \leq C \operatorname{diam}(E)^{-1} \operatorname{diam}\left(\varphi_{\mathrm{ij}}(E)\right) \leq C^{3} \operatorname{diam}\left(X_{\mathrm{i}}\right) \operatorname{diam}\left(X_{\mathrm{j}}\right)
$$

for all $i, j \in I^{*}$. On the other hand,

$$
\operatorname{diam}\left(\varphi_{\mathbf{i}}(E)\right)=\sup _{x, y \in E}\left|\varphi_{\mathbf{i}}(x)-\varphi_{\mathbf{i}}(y)\right| \leq C \operatorname{diam}\left(X_{\mathbf{i}}\right) \sup _{x, y \in E}|x-y|
$$

implies that

$$
\operatorname{diam}\left(X_{\mathbf{i}}\right) \geq C^{-1} \operatorname{diam}(E)^{-1} \operatorname{diam}\left(\varphi_{\mathbf{i}}(E)\right)
$$

for all $i \in I^{*}$. Since

$$
\operatorname{diam}\left(\varphi_{\mathrm{ij}}(E)\right) \geq\left|\varphi_{\mathrm{i}}\left(\varphi_{\mathrm{j}}(x)\right)-\varphi_{\mathrm{i}}\left(\varphi_{\mathrm{j}}(y)\right)\right| \geq C^{-2} \operatorname{diam}\left(X_{\mathrm{i}}\right) \operatorname{diam}\left(X_{\mathrm{j}}\right)|x-y|
$$

for all $x, y \in E$ and $\mathrm{i}, \mathrm{j} \in I^{*}$, we deduce, by (4.8), that

$$
\operatorname{diam}\left(X_{\mathrm{ij}}\right) \geq C^{-1} \operatorname{diam}(E)^{-1} \operatorname{diam}\left(\varphi_{\mathrm{ij}}(E)\right) \geq C^{-3} \operatorname{diam}\left(X_{\mathrm{i}}\right) \operatorname{diam}\left(X_{\mathrm{j}}\right)
$$

for all $i, j \in I^{*}$.

To show (M3), set $M_{n}=\max _{\mathbf{i} \in I^{n}} \operatorname{diam}\left(X_{\mathrm{i}}\right)$ for $n \in \mathbb{N}$ and choose $\dot{i}_{1}, \dot{i}_{2}, \ldots \in I^{\infty}$ such that $M_{n}=\operatorname{diam}\left(X_{\left.\dot{i}_{n}\right|_{n}}\right)$ for every $n \in \mathbb{N}$. By the compactness of $I^{\infty}$, the sequence $\left\{\dot{i}_{n}\right\}_{n \in \mathbb{N}}$ has a converging subsequence. Let $i \in I^{\infty}$ be its limit. Now for each $j \in \mathbb{N}$ there is $n(j) \in \mathbb{N}$ such that $n(j) \geq j$ and $i_{n(j)} \in\left[\left.i\right|_{j}\right]$. Since $\left.i_{n(j)}\right|_{j}=\left.i\right|_{j}$ for all $j \in \mathbb{N}$, we have, using (C1) and (C3),

$$
M_{n(j)}=\operatorname{diam}\left(X_{\left.\mathbf{i}_{n(j)}\right|_{n(j)}}\right) \leq \operatorname{diam}\left(X_{\left.\mathbf{i}_{n(j)}\right|_{j}}\right)=\operatorname{diam}\left(X_{\mathbf{i}_{j}}\right) \rightarrow 0 \quad \text { as } j \rightarrow \infty .
$$

The proof is finished by choosing $j \in \mathbb{N}$ such that $M_{n(j)}<C^{-3}$. 
5. Semiconformal iterated function system. We assume that for each $i \in I$ there is a contractive injection $\varphi_{i}: \Omega \rightarrow \Omega$ defined on an open subset $\Omega$ of $\mathbb{R}^{d}$ and a nonempty closed $X \subset \Omega$ satisfying

$$
\bigcup_{i \in I} \varphi_{i}(X) \subset X
$$

Here the contractivity of $\varphi_{i}$ means that there is a constant $0<s_{i}<1$ such that

$$
\left|\varphi_{i}(x)-\varphi_{i}(y)\right| \leq s_{i}|x-y| \quad \text { for all } x, y \in \Omega .
$$

The collection $\left\{\varphi_{i}: i \in I\right\}$ is then called an iterated function system (IFS). As shown in [15, §3], an elegant application of the Banach fixed point theorem implies the existence of a unique nonempty compact set $E \subset X$ for which

$$
E=\bigcup_{i \in I} \varphi_{i}(E)
$$

Such an $E$ is called the invariant set (for the corresponding IFS). Note that the mappings $\varphi_{i}$ need not be injective to ensure the existence of the invariant set. However, under this additional assumption, Brouwer's domain invariance theorem [6, Theorem IV.7.4] implies that $\varphi_{i}(U)$ is open whenever $U$ is.

Observe that $X$ can be chosen to be a closed neighborhood of $E$. Indeed, fix $0<\varepsilon<\operatorname{dist}\left(E, \mathbb{R}^{d} \backslash \Omega\right.$ ) (if $\Omega=\mathbb{R}^{d}$, any positive $\varepsilon$ will do) and take

$$
X=\{x \in \Omega:|x-a| \leq \varepsilon \text { for some } a \in E\} .
$$

Then (5.1) is a consequence of the easily proven fact that

$$
\operatorname{dist}\left(\varphi_{i}(A), E\right) \leq s_{i} \operatorname{dist}(A, E) \quad \text { for all } A \subset \Omega \text { and } i \in I \text {. }
$$

We say that an IFS is tractable if there exists a compact set $A \subset \Omega$ and a constant $C>0$ such that for each $i \in I^{*}$,

$$
\left|\varphi_{\mathbf{i}}(x)-\varphi_{\mathbf{i}}(y)\right| \leq C \operatorname{diam}\left(\varphi_{\mathrm{i}}(A)\right)|x-y| \quad \text { for } x, y \in A,
$$

and it defines a CMC in this situation, namely $\left\{\varphi_{i}(A): i \in I^{*}\right\}$. The limit set of such a CMC is clearly $E$. Here $\varphi_{i}=\varphi_{i_{1}} \circ \cdots \circ \varphi_{i_{n}}$ for $i=\left(i_{1}, \ldots, i_{n}\right) \in I^{n}$ and $n \in \mathbb{N}$.

LEMMA 5.1. A tractable IFS defines a tractable CMC.

Proof. Choose a compact set $A \subset X$ such that $\left\{\varphi_{i}(A): i \in I^{*}\right\}$ is a CMC. Then

$$
\operatorname{dist}\left(\varphi_{\mathrm{ih}}(A), \varphi_{\mathrm{ik}}(A)\right) \leq C \operatorname{diam}\left(\varphi_{\mathrm{i}}(A)\right) \operatorname{dist}\left(\varphi_{\mathrm{h}}(A), \varphi_{\mathrm{k}}(A)\right),
$$

which implies (3.2) and finishes the proof.

Furthermore, we say that an IFS is semiconformal if the invariant set $E$ has positive diameter and there are constants $0<\underline{s}_{i} \leq \bar{s}_{i}<1$, i $\in I^{*}$, and 
$D \geq 1$ such that $\bar{s}_{\dot{i}} \leq D \underline{s}_{\mathrm{i}}$ for all $\mathrm{i} \in I^{*}$ and

$$
\underline{s}_{\mathrm{i}}|x-y| \leq\left|\varphi_{\mathrm{i}}(x)-\varphi_{\mathrm{i}}(y)\right| \leq \bar{s}_{\mathrm{i}}|x-y| \quad \text { for all } x, y \in \Omega .
$$

For an interesting class of quasiregular mappings which admit uniform control of the distortion with respect to iteration, the reader is referred to [25].

The following lemma shows that a semiconformal IFS defines a semiconformal CMC. The natural question whether the converse holds arises from Lemma 4.1. Sufficient geometric conditions on the limit set for the positive answer to hold are provided in [1]. See also [36, Example 6.2].

LEMmA 5.2. If $\left\{\varphi_{i}: i \in I\right\}$ is a semiconformal IFS, and a compact set $A$ with positive diameter satisfies $\varphi_{i}(A) \subset A$ for every $i \in I$, then $\left\{\varphi_{\mathrm{i}}(A)\right.$ : $\left.i \in I^{*}\right\}$ is a semiconformal CMC. In particular, a semiconformal IFS is tractable. Furthermore, $\left.\varphi_{\mathrm{i}}\right|_{E}, \mathrm{i} \in I^{*}$, are the mappings of Lemma 4.1.

Proof. To use Proposition 4.10, we have to verify (C1)-(C3). Observe first that $(\mathrm{C} 1)$ is clearly satisfied and the positivity of $\operatorname{diam}(E)$ implies $(\mathrm{C} 2)$. Notice also that the sets $\varphi_{\mathrm{i}}(A), \mathrm{i} \in I^{*}$, are compact with positive diameter. Since for fixed $\mathrm{i} \in I^{*}$, we have $\underline{s}_{\mathbf{i}} \operatorname{diam}(A) \leq \operatorname{diam}\left(\varphi_{\mathbf{i}}(A)\right) \leq \bar{s}_{\mathbf{i}} \operatorname{diam}(A)$ by (5.4), it follows that

$$
C^{-1} \operatorname{diam}\left(\varphi_{\mathrm{i}}(A)\right)|x-y| \leq\left|\varphi_{\mathrm{i}}(x)-\varphi_{\mathrm{i}}(y)\right| \leq C \operatorname{diam}\left(\varphi_{\mathrm{i}}(A)\right)|x-y|,
$$

where $C=D \max \left\{\operatorname{diam}(A), \operatorname{diam}(A)^{-1}\right\}$ and $x, y \in A$. Hence, applying (5.2) to (5.5) several times, we get $\operatorname{diam}\left(\varphi_{\mathrm{i}}(A)\right) \leq C s_{i_{1}} \cdots s_{i_{|\mathrm{i}|}}$, which implies (C3). Since (5.5) also yields (C4), that is,

$$
\begin{gathered}
C^{-1} \operatorname{diam}\left(\varphi_{\mathrm{i}}(A)\right) \operatorname{dist}\left(\varphi_{\mathrm{h}}(A), \varphi_{\mathrm{k}}(A)\right) \leq \operatorname{dist}\left(\varphi_{\mathrm{ih}}(A), \varphi_{\mathrm{ik}}(A)\right) \\
\leq C \operatorname{diam}\left(\varphi_{\mathrm{i}}(A)\right) \operatorname{dist}\left(\varphi_{\mathrm{h}}(A), \varphi_{\mathrm{k}}(A)\right)
\end{gathered}
$$

for all $\mathrm{h}, \mathrm{k} \in I^{*}$, we have finished the proof of the first claim.

The second claim follows from (5.5) by recalling that $E$ has positive diameter and satisfies $\varphi_{i}(E) \subset E$ for every $i \in I$. The third claim is immediate since $\left\{\varphi_{\mathrm{i}}(E): \mathrm{i} \in I^{*}\right\}$ is a semiconformal CMC.

We say that an IFS satisfies the open set condition $(O S C)$ if there exists a nonempty open set $U \subset \Omega$ such that

$$
\varphi_{\mathrm{i}}(U) \cap \varphi_{\mathrm{j}}(U)=\emptyset \quad \text { whenever } \mathrm{i} \perp \mathrm{j} .
$$

See [31, Theorem III] for the motivation of the definition. Adapting the terminology from [3], we call any such $U$ a feasible set for the OSC. If there is a feasible set intersecting $E$, we say that the strong $O S C$ is satisfied. As an immediate consequence of the definition, each nonempty open subset and each image $\varphi_{\mathrm{i}}(U)$ of a feasible set $U$ is feasible as well. Thus, using (5.3) repeatedly, we see that the OSC is equivalent to the existence of a feasible set $U \subset X$. Recall that $X$ is a fixed compact $\varepsilon$-neighborhood of the invariant 
set. The next lemma shows that this definition of the OSC is equivalent to the more commonly used one (see $[15, \S 5.2]$ ).

LEMMA 5.3. An IFS satisfies the OSC exactly when there exists a nonempty open set $V \subset X$ such that $\varphi_{i}(V) \subset V$ for all $i \in I$ and $\varphi_{i}(V) \cap \varphi_{j}(V)$ $=\emptyset$ for $i \neq j$. Furthermore, there exists a feasible set intersecting $E$ if and only if there exists a set $V$ as above such that $V \cap E \neq \emptyset$.

Proof. Defining $V=\bigcup_{\mathrm{h} \in I^{*}} \varphi_{\mathrm{h}}(U)$, where $U \subset X$ is a feasible set for the OSC, we clearly have $\varphi_{i}(V) \subset V \subset X$ for all $i \in I$. If $i \neq j$, then

$$
\varphi_{i \mathrm{~h}}(U) \cap \varphi_{j \mathrm{~h}}(U)=\emptyset \quad \text { for every } \mathrm{h} \in I^{*}
$$

and hence

$$
\left(\bigcup_{\mathrm{h} \in I^{*}} \varphi_{i \mathrm{~h}}(U)\right) \cap\left(\bigcup_{\mathrm{h} \in I^{*}} \varphi_{j \mathrm{~h}}(U)\right)=\emptyset .
$$

Noting that the other direction is trivial finishes the proof.

Given IFS, we say that $A \subset \Omega$ is forwards invariant if $\varphi_{i}(A) \subset A$ for all $i \in I$, and backwards invariant if $\varphi_{i}^{-1}(A) \subset A$ for all $i \in I$. For $A \subset \Omega$ we define

$$
F_{A}=\bigcup_{i \perp j} \varphi_{i}^{-1}\left(\varphi_{j}(A)\right)
$$

and for a semiconformal IFS we set

$$
O_{A}=\left\{x \in \Omega: D \operatorname{dist}(x, A)<\operatorname{dist}\left(x, F_{A} \cup\left(\mathbb{R}^{d} \backslash \Omega\right)\right)\right\} .
$$

Here the constant $D \geq 1$ is the same as in the definition of the semiconformal IFS. Observe that $F_{A} \subset \Omega$ is backwards invariant.

Proposition 5.4. Suppose a given IFS is semiconformal. If $U \subset \Omega$ is a feasible set for the $O S C$ then $O_{U} \neq \emptyset$. Furthermore, if there exists a set $A \subset \Omega$ such that $O_{A} \neq \emptyset$ then $O_{A}$ is feasible.

Proof. Let $U \subset \Omega$ be a nonempty open set for which $\varphi_{\mathrm{i}}(U) \cap \varphi_{\mathrm{j}}(U)=\emptyset$ whenever $i \perp j$. It follows that $U \cap F_{U}=\emptyset$ and since $U$ is open, we get $U \subset O_{U}$.

Conversely, it suffices to show that $\varphi_{\mathrm{i}}\left(O_{A}\right) \cap \varphi_{\mathrm{j}}\left(O_{A}\right)=\emptyset$ if $\mathrm{i} \perp \mathrm{j}$. Suppose that there are $i, j \in I^{*}$ and $x, y \in O_{A}$ such that $i \perp j$ and $\varphi_{\mathrm{i}}(x)=\varphi_{\mathrm{j}}(y)=: z$. Observe that the inverse mapping $\varphi_{\mathrm{i}}^{-1}: \varphi_{\mathrm{i}}(\Omega) \rightarrow \Omega$ has a Lipschitz constant $\underline{s}_{i}^{-1}$ for each $i \in I^{*}$. According to Kirszbraun's theorem $[12, \S 2.10 .43]$, there exists a Lipschitz extension $\bar{\varphi}_{i}: \Omega \rightarrow \mathbb{R}^{d}$ of $\varphi_{i}^{-1}$ having the same Lipschitz constant. Since $\bar{\varphi}_{\mathrm{i}}\left(\varphi_{\mathrm{j}}(A)\right) \subset F_{A} \cup\left(\mathbb{R}^{d} \backslash \Omega\right)$ and $x \in O_{A}$, we have 


$$
\begin{aligned}
\operatorname{dist}\left(z, \varphi_{\mathrm{j}}(A)\right) & =\operatorname{dist}\left(\varphi_{\mathrm{i}}(x), \varphi_{\mathrm{j}}(A)\right) \geq \underline{s}_{\mathrm{i}} \operatorname{dist}\left(x, \bar{\varphi}_{\mathrm{i}}\left(\varphi_{\mathrm{j}}(A)\right)\right) \\
& \geq \underline{s}_{\mathrm{i}} \operatorname{dist}\left(x, F_{A} \cup\left(\mathbb{R}^{d} \backslash \Omega\right)\right)>\underline{s}_{\mathrm{i}} D \operatorname{dist}(x, A) \\
& \geq \underline{s}_{\mathrm{i}} D \bar{s}_{\mathrm{i}}^{-1} \operatorname{dist}\left(\varphi_{\mathrm{i}}(x), \varphi_{\mathrm{i}}(A)\right) \geq \operatorname{dist}\left(z, \varphi_{\mathrm{i}}(A)\right)
\end{aligned}
$$

using (5.4). Changing the roles of $i$ and $j$ above, we end up with a contradiction.

We say that a tractable IFS $\left\{\varphi_{i}: i \in I\right\}$ satisfies the ball condition if the corresponding $\mathrm{CMC}\left\{\varphi_{\mathrm{i}}(A): \mathrm{i} \in I^{*}\right\}$ satisfies the (uniform) ball condition. By Lemma 5.2, this defines the ball condition also for a semiconformal IFS. In this case, we may choose $A$ to be the invariant set $E$. Observe that if the IFS is semiconformal and there exists an open set $W \subset \Omega$ such that for each $r>0$ we have

$$
\varphi_{\mathrm{i}}(W) \cap \varphi_{\mathrm{j}}(W)=\emptyset
$$

for any two distinct $i, j \in Z(r)$ then the ball condition is satisfied. See Example 6.5. In particular, the OSC implies the ball condition in the semiconformal case. See also [17, Proposition 3.6]. The following theorem says that, in fact, the ball condition and the strong OSC are equivalent. Example 6.4 shows that this is not true for tractable IFS's.

THEOREM 5.5. A semiconformal IFS satisfies the ball condition exactly when $O_{E} \cap E \neq \emptyset$.

Proof. Let us first prove that the ball condition implies $O_{E} \cap E \neq \emptyset$. Recall that $X$ is the closed $\varepsilon$-neighborhood of $E$. We may further assume that

$$
F:=\bigcup_{i \perp j} \varphi_{i}^{-1}\left(\varphi_{j}(E)\right) \cap X \neq \emptyset,
$$

since $F=\emptyset$ implies $\operatorname{dist}\left(E, F_{E}\right) \geq \varepsilon$, which gives $E \subset O_{E}$. It is now sufficient to find a point $x \in E$ with $\operatorname{dist}(x, F)>0$.

By Theorem 3.5 and Corollary 4.8, there exist $x \in E$ and $\delta>0$ such that

$$
\left|\varphi_{\mathrm{i}}(x)-\varphi_{\mathrm{jh}}(x)\right|>\delta \operatorname{diam}\left(\varphi_{\mathrm{i}}(X)\right)
$$

whenever $\mathrm{i} \perp \mathrm{j}$ and $\mathrm{h} \in I^{*}$. It is easy to see that $\left\{\varphi_{\mathrm{jh}}(x): \mathrm{h} \in I^{*}\right\}$ is dense in $\varphi_{j}(E)$. So, in fact, we have

$$
\operatorname{dist}\left(\varphi_{\mathrm{i}}(x), \bigcup_{i \perp j} \varphi_{j}(E)\right) \geq \delta \operatorname{diam}\left(\varphi_{\mathrm{i}}(X)\right)
$$

for each $i \in I^{*}$, which in turn implies that

$$
\left|\varphi_{\mathrm{i}}(x)-\varphi_{\mathrm{i}}(y)\right| \geq \delta \operatorname{diam}\left(\varphi_{\mathrm{i}}(X)\right)
$$

for each $y \in \varphi_{i}^{-1}\left(\varphi_{j}(E)\right)$ when $i \perp j$. On the other hand, Lemma 5.2 shows 
that there is a constant $C>0$ such that

$$
\left|\varphi_{\mathrm{i}}(x)-\varphi_{\mathrm{i}}(y)\right| \leq C \operatorname{diam}\left(\varphi_{\mathrm{i}}(X)\right)|x-y|
$$

for all $x, y \in X$ and $i \in I^{*}$. Combining the inequalities above gives $|x-y| \geq$ $C^{-1} \delta$ for each $y \in F$ and consequently $\operatorname{dist}(x, F)>0$ as desired.

Since the other direction follows immediately from Proposition 5.4, the proof is finished.

The following proposition generalizes [34, Corollary 2.3] and [33, Corollary 1.2] into the setting of semiconformal IFS's. Although the argument used here is similar to the proof of [33, Corollary 1.2], we give the details for the convenience of the reader.

Proposition 5.6. If a semiconformal IFS satisfies the OSC and $\operatorname{dim}_{H}(E)=d$ then the invariant set $E$ is the closure of its interior.

Proof. As the OSC implies the uniform finite clustering property, we have $P(d)=0$. Hence there exists a constant $c>0$ such that

$$
\sum_{\mathbf{i} \in I^{n}} \underline{s}_{\mathbf{i}}^{d} \geq D^{-d} \operatorname{diam}(X)^{-d} \sum_{\mathbf{i} \in I^{n}} \operatorname{diam}\left(\varphi_{\mathbf{i}}(X)\right)^{d} \geq c
$$

(see the defining equation (3.1) and Lemma 2.1). Choose the forwards invariant feasible set $V \subset X$ as in Lemma 5.3 and consider the set

$$
T=V \backslash \bigcup_{i \in I} \varphi_{i}(V) \text {. }
$$

The facts that $\varphi_{\mathrm{i}}(T) \subset \varphi_{\mathrm{i}}(V)$ and $\varphi_{\mathrm{i}}(T) \cap \varphi_{\mathrm{ij}}(V)=\emptyset$ for all $\mathrm{i} \in I^{*}$ and $\mathrm{j} \in I^{*}$ easily lead to the conclusion that $\varphi_{\mathrm{i}}(T) \cap \varphi_{\mathrm{j}}(T)=\emptyset$ whenever $\mathrm{i} \neq \mathrm{j}$. Furthermore, since $\varphi_{i}(T) \subset X$ for each $i \in I^{*}$, we have

$$
\begin{aligned}
\infty>\mathcal{H}^{d}(X) & \geq \mathcal{H}^{d}\left(\bigcup_{i \in I^{*}} \varphi_{\mathrm{i}}(T)\right)=\sum_{n \in \mathbb{N}} \sum_{i \in I^{n}} \mathcal{H}^{d}\left(\varphi_{\mathrm{i}}(T)\right) \\
& \geq \mathcal{H}^{d}(T) \sum_{n \in \mathbb{N}} \sum_{\mathbf{i} \in I^{n}} \underline{s}_{i}^{d} .
\end{aligned}
$$

Now (5.6) and (5.7) together imply that $\mathcal{H}^{d}(T)=0$. This in turn shows that the set

$$
V \backslash \overline{\bigcup_{i \in I} \varphi_{i}(V)}=V \backslash \bigcup_{i \in I} \varphi_{i}(\bar{V})
$$

is empty, being an open set with zero measure. Here $\bar{A}$ stands for the closure of $A$. This means that $\bar{V}=\bigcup_{i \in I} \varphi_{i}(\bar{V})$, giving $E=\bar{V}$ by the uniqueness of the invariant set.

A similitude IFS, introduced in [15], is the most obvious example of a semiconformal IFS. Suppose that for each $i \in I$ there is a mapping $\varphi_{i}$ : 
$\mathbb{R}^{d} \rightarrow \mathbb{R}^{d}$ and a constant $0<s_{i}<1$ such that

$$
\left|\varphi_{i}(x)-\varphi_{i}(y)\right|=s_{i}|x-y| \quad \text { for all } x, y \in \mathbb{R}^{d} .
$$

Now for a closed ball $B$ centered at the origin, we have $\varphi_{i}(B) \subset B$ for all $i \in I$ provided that the radius of $B$ is chosen large enough. The collection $\left\{\varphi_{i}: i \in I\right\}$ is then an IFS and we call it a similitude IFS.

The following proposition is slightly more general than [3, Theorem 1].

Proposition 5.7. Given a similitude IFS, the set $O_{A}$ is forwards invariant and feasible for the $O S C$ provided that $O_{A} \neq \emptyset$ and $A \subset X$ is forwards invariant.

Proof. According to Proposition 5.4, it suffices to show that $\varphi_{i}\left(O_{A}\right) \subset$ $O_{A}$ for all $i \in I$. Assume on the contrary that there exist $i \in I$ and $x \in O_{A}$ such that $\varphi_{i}(x) \notin O_{A}$, that is,

$$
D \operatorname{dist}\left(\varphi_{i}(x), A\right) \geq \operatorname{dist}\left(\varphi_{i}(x), F_{A}\right) .
$$

Notice that here $D$ can be chosen to be 1 . Therefore, since $A \subset \varphi_{i}^{-1}(A)$ and $\varphi_{i}^{-1}\left(F_{A}\right) \subset F_{A}$ for every $i \in I$, we obtain

$$
\begin{aligned}
\operatorname{dist}\left(x, F_{A}\right) & >D \operatorname{dist}(x, A) \geq D \operatorname{dist}\left(x, \varphi_{i}^{-1}(A)\right) \\
& =s_{i}^{-1} D \operatorname{dist}\left(\varphi_{i}(x), A\right) \geq s_{i}^{-1} \operatorname{dist}\left(\varphi_{i}(x), F_{A}\right) \\
& =\operatorname{dist}\left(x, \varphi_{i}^{-1}\left(F_{A}\right)\right) \geq \operatorname{dist}\left(x, F_{A}\right) .
\end{aligned}
$$

This contradiction finishes the proof.

The following corollary summarizes the main implications shown for a semiconformal IFS. Notice that the topological pressure here is well defined via Lemma 5.2 as it does not depend on the choice of the corresponding forwards invariant set.

COROLlary 5.8. For a semiconformal IFS, the following conditions are equivalent:

(1) The ball condition.

(2) The open set condition.

(3) The strong open set condition.

(4) $\mathcal{H}^{t}(E)>0$, where $t$ is the zero of the topological pressure.

6. Examples. In this last section, we illustrate the theory by providing several examples. We begin by showing that the uniform finite clustering property does not imply the bounded overlapping property.

Example 6.1. The standard Cantor $1 / 3$-set $E$ can be defined as the invariant set of the similitude IFS formed by the mappings

$$
\varphi_{0}(x)=\frac{1}{3} x, \quad \varphi_{1}(x)=\frac{1}{3} x+\frac{2}{3}
$$


on $\mathbb{R}$. It is well known that $\mathcal{H}^{t}(E)=1$, where $t=\log 2 / \log 3$ (see $[9$, Theorem 1.14]). Consider now the $\operatorname{CMC}\left\{\varphi_{\mathrm{i}}(X): \mathrm{i} \in I^{*}\right\}$, where $X=[0,3]$ and $I=\{0,1\}$. It is tractable by Lemma 5.1. The positivity of $\mathcal{H}^{t}(E)$ implies the uniform finite clustering property by Theorem 3.9. However, as $1 \in \varphi_{0}(X)$ and $\varphi_{1}(1)=1$, we infer by induction that $1 \in \varphi_{1^{k}}(X)$ for every $k \in \mathbb{N}$, where $1^{k}=(1, \ldots, 1) \in I^{k}$ for each $k$. Since the infinite set $\left\{1^{k} 0: k \in \mathbb{N}\right\}$ is incomparable, we conclude that the bounded overlapping property is not satisfied.

EXAMPLE 6.2. We now give a CMC which shows that the assumption on the relative positions of the sets $X_{i}$ in the last claim of Proposition 3.2 is indispensable. Moreover, it is also an example of a nontractable CMC. Using the mappings $\varphi_{i}, i \in I^{*}$, from the previous example, set

$$
X_{0}=[0,1] \times[0,1], \quad X_{1}=[0,1] \times[-1,0],
$$

and for $j \in I$ and $i \in I^{*}$,

$$
X_{j \mathrm{i}}= \begin{cases}\varphi_{\mathrm{i}}([0,1]) \times\left[0,3^{-|\mathrm{i}|}\right] & \text { if } j=0, \\ \varphi_{\mathrm{i}}([0,1]) \times\left[-3^{-|\mathrm{i}|}, 0\right] & \text { if } j=1 .\end{cases}
$$

The CMC determined by these squares obviously has the limit set $E=$ $E_{x} \times\{0\} \subset \mathbb{R}^{2}$, where $E_{x} \subset \mathbb{R}$ is the standard Cantor $1 / 3$-set. It is equally obvious that the uniform ball condition is satisfied, which, according to Theorems 3.7 and 3.5 and Remark 3.8, implies that the measure $m$ of Proposition 3.2 is proportional to $\left.\mathcal{H}^{t}\right|_{E}$, where $t=\log 2 / \log 3$ as in the previous example. Consequently, $m(J)>0$ whenever $J$ is one of the line segments $\varphi_{\mathrm{i}}([0,1]) \times\{0\}, \mathrm{i} \in I^{*}$. In particular,

$$
m\left(X_{\mathrm{i}} \cap X_{\mathrm{j}}\right)>0
$$

for any incomparable symbols $i$ and $j$ satisfying $\left.i\right|_{1} \neq\left. j\right|_{1}$ and $\sigma(i)=\sigma(j)$. We have thus shown that the measure $m$ is not $t$-semiconformal. On the other hand, Lemma 3.6 implies that the bounded overlapping property is satisfied, as clearly $X_{i} \cap E=\pi([i])$ for each $i \in I^{*}$. Therefore, the extra assumption in Proposition 3.2 is really needed.

Furthermore, this CMC is not tractable. This can be deduced from the fact that

$$
\operatorname{dist}\left(X_{0 \mathrm{i}}, X_{1 \mathrm{i}}\right)=0
$$

but

$$
\operatorname{dist}\left(X_{00 \mathrm{i}}, X_{01 \mathrm{i}}\right) \geq \operatorname{dist}\left(X_{00}, X_{01}\right)=\frac{1}{3}
$$

for every $i \in I^{*}$.

EXAMPLE 6.3. We now define a class of nonsemiconformal tractable IFS's. Suppose $I$ is a finite set and for each $i \in I$ there is a mapping 
$\varphi_{i}: \mathbb{R}^{2} \rightarrow \mathbb{R}^{2}$ such that

$$
\varphi_{i}(x, y)=\left(a_{i} x+c_{i}, b_{i} y+d_{i}\right),
$$

where $0<b_{i}<a_{i}<1$ and $c_{i}, d_{i} \geq 0$. Defining $a_{\mathrm{i}}=a_{i_{1}} \cdots a_{i_{n}}$ and $b_{\mathrm{i}}=$ $b_{i_{1}} \cdots b_{i_{n}}$ for each $\mathrm{i}=\left(i_{1}, \ldots, i_{n}\right) \in I^{n}$ and $n \in \mathbb{N}$, we have

$$
\begin{aligned}
& \sup _{\left(x_{1}, y_{1}\right) \neq\left(x_{2}, y_{2}\right)} \frac{\left|\varphi_{\mathbf{i}}\left(x_{1}, y_{1}\right)-\varphi_{\dot{\mathrm{i}}}\left(x_{2}, y_{2}\right)\right|}{\left|\left(x_{1}, y_{1}\right)-\left(x_{2}, y_{2}\right)\right|}=a_{\dot{i}}, \\
& \inf _{\left(x_{1}, y_{1}\right) \neq\left(x_{2}, y_{2}\right)} \frac{\left|\varphi_{\mathbf{i}}\left(x_{1}, y_{1}\right)-\varphi_{\dot{i}}\left(x_{2}, y_{2}\right)\right|}{\left|\left(x_{1}, y_{1}\right)-\left(x_{2}, y_{2}\right)\right|}=b_{\mathbf{i}}
\end{aligned}
$$

for all $\mathrm{i} \in I^{*}$. It is clear that $b_{\mathrm{i}} / a_{\mathrm{i}} \rightarrow 0$ as $|\mathrm{i}| \rightarrow \infty$, showing that the IFS $\left\{\varphi_{i}: i \in I\right\}$ is not semiconformal. However, by choosing $L=1+$ $\max _{i \in I}\left\{c_{i}, d_{i}\right\} /\left(1-\max _{i \in I} a_{i}\right)$ and $X=[0, L]^{2}$, we get $\varphi_{i}(X) \subset X$ for every $i \in I$ and

$$
a_{\mathrm{i}} L \leq \operatorname{diam}\left(\varphi_{\mathrm{i}}(X)\right) \leq \sqrt{2} a_{\mathrm{i}} L
$$

for each $i \in I^{*}$. The collection $\left\{\varphi_{i}(X): i \in I^{*}\right\}$ is thus a CMC, and consequently the IFS $\left\{\varphi_{i}: i \in I\right\}$ is tractable.

According to Corollary 3.10, this CMC satisfies the (uniform) ball condition if and only if $0<\mathcal{H}^{t}(E)<\infty$, where $E$ is the limit set and $\sum_{i \in I} a_{i}^{t}=1$. For related dimension results, see [29], [14], and [13].

Observe also that choosing, for example, $I=\{0,1\}, 0<b_{0}=b_{1} \leq a_{0}=$ $a_{1} \leq 1 / 2, c_{1}>0$, and $d_{0} \geq 0=c_{0}=d_{1}$, it is straightforward to see that the ball condition is automatically satisfied.

EXAMPLE 6.4. Recall that by Corollary 5.8, a semiconformal IFS satisfies the OSC if and only if it satisfies the ball condition. We now show that for a tractable IFS this equivalence is not necessarily true.

In Example 6.3, choose $I=\{0,1\}, 0<b_{0}=b_{1}<a_{0}=a_{1} \leq 1 / 2$, $d_{0}>0$, and $c_{0}=c_{1}=d_{1}=0$. It is clear that this tractable IFS satisfies the OSC. It can be seen by a straightforward calculation that the uniform finite clustering property fails, implying that the uniform ball condition does not hold. Alternatively, it follows from the observations done in Example 6.3 that the invariant set $E$ has Hausdorff dimension $-\log 2 / \log a_{0}$ provided that the ball condition is satisfied. However, since $E$ is clearly a subset of $\{0\} \times \mathbb{R}$ having Hausdorff dimension $-\log 2 / \log b_{0}$, this cannot be the case.

We do not know if there exists a tractable IFS satisfying the ball condition but not the OSC.

EXAMPLE 6.5. At first glance, it seems that for a semiconformal IFS the OSC (especially via Lemma 5.3) is easier to check than the ball condition. However, there are cases when it is much more convenient to consider the ball condition than the OSC. We now consider a familiar self-similar set having this property. 
We identify $\mathbb{R}^{2}$ and $\mathbb{C}$ for notational simplicity and we set $\eta=1 / 2+i / 2$. Let $I=\{0,1\}$ and let $\varphi_{0}, \varphi_{1}$ be the similitudes given by the equations

$$
\varphi_{0}(z)=\eta z, \quad \varphi_{1}(z)=\bar{\eta} z+\eta,
$$

where $z \in \mathbb{C}$ and $\bar{\eta}=1 / 2-i / 2$ is the complex conjugate of $\eta$. Notice that the contraction ratio of both mappings is $1 / \sqrt{2}$. The invariant set $E$ of the IFS $\left\{\varphi_{0}, \varphi_{1}\right\}$ is the well known Lévy's dragon (see [24]). Since $\mathcal{H}^{2}(E)>0$, it follows from [34, Theorem 2.1] that the OSC is satisfied and hence by [34, Corollary 2.3 and its proof] and Lemma 5.3, we conclude that a nonempty open set $U$ is feasible only if $U \subset E$. Because of the intricate structure of the Lévy's dragon, such an open set is, a priori, virtually impossible to find. However, it is straightforward to find an open set $W \subset \mathbb{C}$ satisfying

$$
\varphi_{\mathrm{i}}(W) \cap \varphi_{\mathrm{j}}(W)=\emptyset \quad \text { whenever }|\mathrm{i}|=|\mathrm{j}| \text { and } \mathrm{i} \neq \mathrm{j},
$$

from which the ball condition follows for any corresponding semiconformal CMC. This can be done by choosing $W$ to be the interior of the rightangled triangle $\triangle=\operatorname{conv}\{0,1, \eta\}$ and looking at the images $\varphi_{\mathbf{i}}(W)$ for $i \in I^{n}$ with fixed $n$. The calculations for the disjointness of the images are straightforward since the vertices $\varphi_{i}(0)$ and $\varphi_{i}(1)$ of $\varphi_{i}(\triangle)$ belong to the point grid $H_{n}=\left\{\eta^{n}(k+i l): k, l \in \mathbb{Z}\right\}$ whereas $\varphi_{\mathrm{i}}(\eta) \in H_{n+1}$, and $\varphi_{\mathrm{i}}(\triangle)=\varphi_{\mathrm{j}}(\triangle)$ only if $\varphi_{\mathrm{i}}(0)=\varphi_{\mathrm{j}}(0)$ and $\varphi_{\mathrm{i}}(1)=\varphi_{\mathrm{j}}(1)$. See [7, p. 222] for an illustration and apply the calculations done in the appendix of [20]. Notice that the set $W$ above is not feasible since $W \not \subset E$.

EXAMPLE 6.6. We now note that any conformal IFS is semiconformal. Suppose $I$ is a finite set and for each $i \in I$ there is a contractive $C^{1+\varepsilon}$ conformal mapping $\varphi_{i}: \Omega \rightarrow \Omega$ defined on an open set $\Omega \subset \mathbb{R}^{d}$. If there exists a nonempty closed $X \subset \Omega$ satisfying

$$
\bigcup_{i \in I} \varphi_{i}(X) \subset X
$$

the collection $\left\{\varphi_{i}: i \in I\right\}$ is an IFS and we call it a conformal IFS. We deduce from the well known bounded distortion principle that each conformal IFS is semiconformal. See, for example, [28, Remark 2.3]. Observe that the converse does not necessarily hold. For example, the semiconformal IFS constructed in [18, Example 2.1] is not conformal.

EXAMPLE 6.7. Observe that any IFS bi-Lipschitz conjugate to a conformal IFS is semiconformal. Although the bi-Lipschitz conjugacy preserves positivity and finiteness of the Hausdorff measure, the following example is of special interest as it emphasizes that to prove Corollary 5.8 we do not need differentiable mappings.

Let $D^{\prime} \subset[0,1]^{2}$ be the graph of a nondecreasing continuous function $F:[0,1] \rightarrow[0,1]$ satisfying $F(0)=0$ and $F(1)=1$. A well known nondiffer- 
entiable example of this kind of function is $\left.x \mapsto \mathcal{H}^{t}\right|_{E}([0, x])$, where $E$ is the $1 / 3$-Cantor set and $t=\log 2 / \log 3$. In this case, $D^{\prime}$ is known as the devil's stairs. We set $D=D^{\prime} \cup\{(x, x):|x|>1\}, L=\{(x, x): x \in \mathbb{R}\}$, and $\operatorname{proj}_{L}$ to be the orthogonal projection onto $L$. The mapping $f=\left(\left.\operatorname{proj}_{L}\right|_{D}\right)^{-1}$ : $L \rightarrow D$ is clearly bi-Lipschitz and defining a mapping $g: \mathbb{R}^{2} \rightarrow \mathbb{R}^{2}$ by setting $g(x)=x+f\left(\operatorname{proj}_{L}(x)\right)-\operatorname{proj}_{L}(x)$ for each $x \in \mathbb{R}^{2}$, the reader can easily see that also $g$ is bi-Lipschitz.

Since the line segment $L \cap[0,1]^{2}$ is clearly the invariant set of the similitude IFS $\left\{\varphi_{i}: i \in I\right\}$, where

$$
\varphi_{i}(x, y)=\left(\frac{1}{N} x+\frac{i-1}{N}, \frac{1}{N} y+\frac{i-1}{N}\right)
$$

and $I=\{1, \ldots, N\}$, the set $D^{\prime}=g\left(L \cap[0,1]^{2}\right)$ is the invariant set of a semiconformal IFS $\left\{g \circ \varphi_{i} \circ g^{-1}: i \in I\right\}$. Here $N \in \mathbb{N}$ is chosen so large that the mappings $g \circ \varphi_{i} \circ g^{-1}$ are contractions.

The devil's stairs are also an example of a semiconformal IFS which is not conformal (see [16, Theorem 2.1]).

Example 6.8. Defining, for $A \subset \mathbb{R}^{d}, x \in \mathbb{R}^{d}$, and $r>0$,

$$
\begin{aligned}
& \operatorname{por}(A, x, r) \\
& \quad=\sup \left\{\varrho \geq 0: \text { there is } z \in \mathbb{R}^{d} \text { such that } B(z, \varrho r) \subset B(x, r) \backslash A\right\},
\end{aligned}
$$

we say that a bounded set $A \subset \mathbb{R}^{d}$ is uniformly porous if there are $\varrho>0$ and $r_{0}>0$ such that $\operatorname{por}(A, x, r) \geq \varrho$ for all $x \in A$ and $0<r<r_{0}$. The notion of porosity has arisen from the study of dimensional estimates related to the boundary behavior of various mappings.

Following the proof of [19, Theorem 4.1], we notice that a uniformly porous set is contained in a limit set $E$ of a semiconformal CMC satisfying the uniform ball condition such that $\operatorname{dim}_{\mathrm{M}}(E) \leq d-c \varrho^{d}$ (see Theorem 3.7).

\section{References}

[1] P. Alestalo, D. A. Trotsenko, and J. Väisälä, The linear extension property of biLipschitz mappings, Sibirsk. Mat. Zh. 44 (2003), 1226-1238 (in Russian).

[2] C. Bandt and S. Graf, Self-similar sets \%. A characterization of self-similar fractals with positive Hausdorff measure, Proc. Amer. Math. Soc. 114 (1992), 995-1001.

[3] C. Bandt, N. V. Hung, and H. Rao, On the open set condition for self-similar fractals, ibid. 134 (2006), 1369-1374.

[4] L. Barreira, A non-additive thermodynamic formalism and applications to dimension theory of hyperbolic dynamical systems, Ergodic Theory Dynam. Systems 16 (1996), 871-927.

[5] R. Bowen, Equilibrium States and the Ergodic Theory of Anosov Diffeomorphisms, Lecture Notes in Math. 470, Springer, 1975.

[6] A. Dold, Lectures on Algebraic Topology, Springer, Berlin, 1972. 
[7] G. A. Edgar, Classics on Fractals, Addison-Wesley, New York, 1993.

[8] K. Falconer, Dimensions and measures of quasi self-similar sets, Proc. Amer. Math. Soc. 106 (1989), 543-554.

[9] —, Geometry of Fractal Sets, Cambridge Univ. Press, Cambridge, 1985.

[10] —, Techniques in Fractal Geometry, Wiley, 1997.

[11] A. H. Fan and K.-S. Lau, Iterated function system and Ruelle operator, J. Math. Anal. Appl. 231 (1999), 319-344.

[12] H. Federer, Geometric Measure Theory, Springer, Berlin, 1969.

[13] D.-J. Feng and Y. Wang, A class of self-affine sets and self-affine measures, J. Fourier Anal. Appl. 11 (2005), 107-124.

[14] D. Gatzouras and S. P. Lalley, Hausdorff and box dimensions of certain self-affine fractals, Indiana Univ. Math. J. 41 (1992), 533-568.

[15] J. E. Hutchinson, Fractals and self-similarity, ibid. 30 (1981), 713-747.

[16] A. Käenmäki, On the geometric structure of the limit set of conformal iterated function systems, Publ. Mat. 47 (2003), 133-141.

[17] -, On natural invariant measures on generalised iterated function systems, Ann. Acad. Sci. Fenn. Math. 29 (2004), 419-458.

[18] —, Geometric rigidity of a class of fractal sets, Math. Nachr. 279 (2006), 179-187.

[19] A. Käenmäki and V. Suomala, Nonsymmetric conical upper density and k-porosity, preprint, 2004.

[20] J. Kigami, Hausdorff dimensions of self-similar sets and shortest path metrics, J. Math. Soc. Japan, 47 (1995), 381-404.

[21] S. P. Lalley, The packing and covering functions of some self-similar fractals, Indiana Univ. Math. J. 37 (1988), 699-710.

[22] K.-S. Lau, H. Rao, and Y.-L. Ye, Corrigendum: Iterated function system and Ruelle operator, J. Math. Anal. Appl. 262 (2001), 446-451.

[23] K.-S. Lau and Y. Xu, On the boundary of attractors with non-void interior, Proc. Amer. Math. Soc. 128 (2000), 1761-1768.

[24] P. Lévy, Les courbes planes ou gauches et les surfaces composées de parties semblables au tout, J. École Polytechnique 7 (1938), 227-291.

[25] G. Martin, V. Mayer, and K. Peltonen, The generalized Lichnerowicz problem: Uniformly quasiregular mappings and space forms, Proc. Amer. Math. Soc. 134 (2006), 2091-2097.

[26] M. Martín and P. Mattila, On the parametrization of fractal sets, ibid. 128 (2000), 2641-2648.

[27] P. Mattila, Geometry of Sets and Measures in Euclidean Spaces: Fractals and Rectifiability, Cambridge Univ. Press, Cambridge, 1995.

[28] R. D. Mauldin and M. Urbański, Dimensions and measures in infinite iterated function systems, Proc. London Math. Soc. 73 (1996), 105-154.

[29] C. T. McMullen, The Hausdorff dimension of general Sierpiński carpets, Nagoya Math. J. 96 (1984), 1-9.

[30] M. Morán, Dynamical boundary of a self-similar set, Fund. Math. 160 (1999), 1-14.

[31] P. A. P. Moran, Additive functions of intervals and Hausdorff measure, Proc. Cambridge Philos. Soc. 42 (1946), 15-23.

[32] M. E. Munroe, Introduction to Measure and Integration, Addison-Wesley, Cambridge, MA, 1953.

[33] Y. Peres, M. Rams, K. Simon, and B. Solomyak, Equivalence of positive Hausdorff measure and the open set condition for self-conformal sets, Proc. Amer. Math. Soc. 129 (2001), 2689-2699.

[34] A. Schief, Separation properties for self-similar sets, ibid. 122 (1994), 111-115. 
[35] T. Szarek and S. Wędrychowicz, The OSC does not imply the SOSC for infinite iterated function systems, ibid. 133 (2005), 437-440.

[36] J. Väisälä, M. Vuorinen, and H. Wallin, Thick sets and quasisymmetric maps, Nagoya Math. J. 135 (1994), 121-148.

[37] P. Walters, An Introduction to Ergodic Theory, Springer, New York, 1982.

[38] M. S. Waterman, Remarks on invariant measures for number theoretic transformations, Monatsh. Math. 79 (1975), 157-163.

[39] R. L. Wheeden and A. Zygmund, Measure and Integral. An Introduction to Real Analysis, Dekker, New York, 1977.

[40] Y.-L. Ye, Separation properties for self-conformal sets, Studia Math. 152 (2002), $32-44$.

Department of Mathematics and Statistics

P.O. Box 35 (MaD)

FI-40014 University of Jyväskylä, Finland

E-mail: antakae@maths.jyu.fi

vilppola@maths.jyu.fi

Received 7 August 2007;

in revised form 19 March 2008 